TITLE:

\title{
Ginzburg-Landau theory of solvation in polar fluids: Ion distribution around an interface
}

$\operatorname{AUTHOR}(S)$ :

Onuki, A

CITATION:

Onuki, A. Ginzburg-Landau theory of solvation in polar fluids: Ion

distribution around an interface. Physical Review E 2006, 73(2): 021506.

ISSUE DATE:

2006-02

URL:

http://hdl.handle.net/2433/49914

RIGHT:

Copyright 2006 American Physical Society 
PHYSICAL REVIEW E 73, 021506 (2006)

\title{
Ginzburg-Landau theory of solvation in polar fluids: Ion distribution around an interface
}

\author{
Akira Onuki \\ Department of Physics, Kyoto University, Kyoto 606-8502, Japan
}

(Received 17 October 2005; published 21 February 2006)

\begin{abstract}
We present a Ginzburg-Landau theory of solvation of ions in polar binary mixtures. The solvation free energy arising from the ion-dipole interaction can strongly depend on the composition and the ion species. Most crucial in phase separation is then the difference in the solvation free energy between the two phases, which is the origin of the Galvani potential difference known in electrochemistry. We also take into account an image potential acting on each ion, which arises from inhomogeneity in the dielectric constant and is important close to an interface at very small ion densities. Including these solvation and image interactions, we calculate the ion distributions and the electric potential around an interface with finite thickness. In particular, on approaching the critical point, the ion density difference between the two phases becomes milder. The critical temperature itself is much shifted even by a small amount of ions. We examine the surface tension in the presence of ions in various cases.
\end{abstract}

DOI: 10.1103/PhysRevE.73.021506

PACS number(s): 82.45.Gj, 61.20.Qg, 64.70.Ja, 68.03.Cd

\section{INTRODUCTION}

Charge effects have long been discussed extensively in electrolytes, polyelectrolytes, gels, DNA, and so on [1,2]. In most of the physics literature, charged particles interact via the Coulombic potential in a fluid with a homogeneous dielectric constant $\varepsilon$. In the chemistry literature, on the other hand, attention has been paid to the microscopic molecular interactions between an ion and the surrounding solvent molecules $[3,4]$, which give rise to a chemical potential contribution of ions dependent on the solvent density (composition for mixtures) and the temperature $T$. For a microscopic ion, such as $\mathrm{Na}^{+}$or $\mathrm{Cl}^{-}$in a polar fluid, it is the so-called solvation free energy (hydration free energy in water or aqueous solutions) per ion arising from the ion-dipole interaction under the influence of hydrogen bonding $[5,6]$. In this paper, it will be called the solvation chemical potential and will be written as $\mu_{\text {sol }}^{i}$ dependent on the ion species $i$. It is a crucial quantity in understanding the degree of solubility of ions in polar solvents $[3,4]$. Since $\mu_{\mathrm{sol}}^{i}$ strongly depends on the solvent density or composition and can much exceed $k_{B} T$, it should play various important roles particularly in the formation of inhomogeneous structures, which has been one of the main subjects in soft matter physics.

As a classic example, the Born theory [7] takes into account polarization around an ion using the linear dielectric constant $\varepsilon$ and gives the solvation free energy in the form,

$$
\left(\mu_{\mathrm{sol}}^{i}\right)_{\mathrm{Born}}=Z^{2} e^{2} / 2 \varepsilon R_{\mathrm{ion}}^{i},
$$

where $Z e$ is the ion charge and $R_{\text {ion }}^{i}$ is the microscopic ion radius (of order $1 \AA$ for $\mathrm{Na}^{+}$) [3,8]. For water, $\varepsilon$ is of order 1 in gas and of order 100 in liquid at room temperatures, so its density dependence is very strong [9]. For mixtures of two fluid components, $A$ and $B, \varepsilon$ changes from the dielectric constant $\varepsilon_{B}$ of the less polar component to that $\varepsilon_{A}$ of the more polar component with increasing the volume fraction $\phi$ of the more polar component $[4,10]$. However, it is well known that the Born formula neglects electrostriction $[3,11]$ and nonlinear dielectric saturation [12-14] in the vicinity of an ion and is in many cases an overestimation. For mixtures, the former effect means that molecules of the more polar component accumulate around an ion to form the solvation shell $[3,4]$. The latter effect means that the effective dielectric constant in the vicinity of an ion should be smaller than in the bulk. Nevertheless, the Born formula demonstrates strong dependence of $\mu_{\mathrm{sol}}^{i}$ on the solvent density or composition.

Let a fluid be phase separated into a highly polar phase $\alpha$ and a less polar phase $\beta$. Generally, $\mu_{\text {sol }}^{i}$ takes different values in the two bulk phases and these values depend on the ion species $i\left(\mathrm{Na}^{+}\right.$or $\mathrm{Cl}^{-}$, for example), so they are written as $\mu_{\mathrm{sol}}^{i \alpha}$ and $\mu_{\mathrm{sol}}^{i \beta}$. In the electrochemistry literature [15-19], the chemical-potential difference

$$
\Delta \mu_{\alpha \beta}^{i}=\mu_{\mathrm{sol}}^{i \beta}-\mu_{\mathrm{sol}}^{i \alpha}
$$

between the two phases is called the standard Gibbs transfer energy (usually per mole, but per ion in this paper). Using data of $\Delta \mu_{\alpha \beta}^{i}$ on water+nitrobenzene [17] (with $\alpha$ being a water-rich phase), we estimate $\Delta \mu_{\alpha \beta}^{i} / k_{B} T$ as 13.6 for $\mathrm{Na}^{+}$, 10.6 for $\mathrm{K}^{+}, 11.3$ for $\mathrm{Br}^{-}$, and 7.46 for $\mathrm{I}^{-}$, where we set $T$ $=300 \mathrm{~K}$. Notice that if $\Delta \mu_{\alpha \beta}^{i} \gg k_{B} T$, there arises a large difference in the ion density between the two phases. Furthermore, since $\Delta \mu_{\alpha \beta}^{i}$ depends on the ion species, there can be an electric double layer at the interface, which then leads to an electric potential difference across the interface, called the Galvani potential difference. We also note that $\Delta \mu_{\alpha \beta}^{i}$ is a parameter dramatically influencing the nucleation process when a small amount of ions are doped in a metastable polar fluid [20,21]. Recently, $\Delta \mu_{\alpha \beta}^{i}$ was calculated between gaseous water (phase $\beta$ ) and liquid water (phase $\alpha$ ), which is much larger than $k_{B} T$ far below the critical point [22].

It has also long been known that the surface tension of a water-air interface increases with increasing the salt density [23-30]. The ion density nearly vanishes in the air region, obviously due to very large $\Delta \mu_{\alpha \beta}^{i} / k_{B} T$. Here, phase $\alpha$ is salty water and phase $\beta$ is air. It has been argued that ions in water should be repelled away from the interface by the image 
charges in air $[24,25]$. This leads to a depletion of the ion density near the interface and an increase of the surface tension,

$$
\Delta \gamma=k_{B} T n_{\text {salt }} \lambda_{s},
$$

where $n_{\text {salt }}$ is the salt density in the bulk water and $\lambda_{s}$ is the effective thickness of the ion-free layer. This is in marked contrast to the role of surfactant molecules, which serve to decrease the surface tension [31]. For example, $\lambda_{s}$ is about 6 $\AA$ for $\mathrm{NaCl}$ at room temperatures [29]. The image interaction comes into play in $\lambda_{s}$ at low salt densities, where $\Delta \gamma$ itself is small, however. Here, we mention microscopic calculations of the ion distributions at a water-air interface [6] (see discussions in Sec. III).

We stress that the strong density or composition dependence of $\mu_{\text {sol }}^{i}$ gives rise to a number of unexplored physical effects, particularly in two-phase states. As a paper in this direction, we will examine the ion distributions around an interface in the scheme of Ginzburg-Landau theory $[22,32,33]$. In Sec. II, we will present our scheme accounting for electrostatics with inhomogeneous $\varepsilon$, solvation effects, and image forces. In Sec. III, we will explain our numerical scheme and show profiles of the electric field in a parallelplate capacitor. In Sec. IV, we will present a number of general relations and show numerical results of the interface structure. In Appendix A, we will summarize the calculations of the structure factor of the composition fluctuations and the effective interaction potentials among the ions in near-critical one-phase states.

\section{GINZBURG-LANDAU THEORY}

Let us consider a polar binary mixture with a small amount of salt composed of two species of ions. The volume fraction of the more polar component $A$ will be written as $\phi(\boldsymbol{r})$. The ion densities are $n_{1}(\boldsymbol{r})$ and $n_{2}(\boldsymbol{r})$. In the continuum limit, $\phi, n_{1}$, and $n_{2}$ are smooth variables coarse-grained on the microscopic level, and we will set up the GinzburgLandau free energy $F$ as a functional of these variables. We will assume that the ion densities are small and will neglect formation of dipole pairs [2,34]. We divide $F$ into three parts as

$$
F=F_{e}+F_{\mathrm{ch}}+F_{\mathrm{im}}
$$

and will explain each term in the following.

\section{A. Electrostatics}

We assume that the dielectric constants $\varepsilon_{A}$ and $\varepsilon_{B}$ of the two components are significantly different and concentration dependence of the dielectric constant of the mixture may be expressed as [10]

$$
\varepsilon(\phi)=\varepsilon_{0}+\varepsilon_{1} \phi,
$$

where $\varepsilon_{0}$ and $\varepsilon_{1}$ are positive constants with [35]

$$
\varepsilon_{0}=\varepsilon_{B}, \quad \varepsilon_{1}=\varepsilon_{A}-\varepsilon_{B}>0 .
$$

Empirically, $\varepsilon$ can be expressed roughly as a linear function of the concentration in many mixtures investigated so far

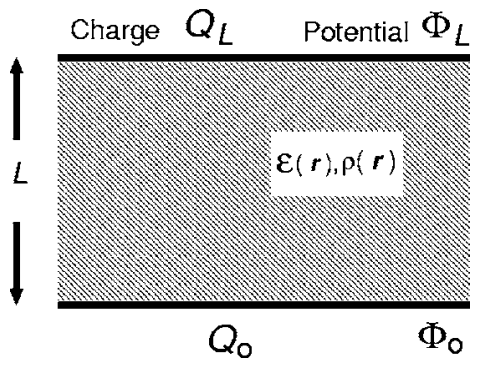

FIG. 1. Ionic fluid between parallel metal plates with spacedependent dielectric constant $\varepsilon$ and charge density $\rho$. Surface charges $Q_{L}$ and $Q_{0}$ are fixed.

$[4,10]$. Hereafter, $\boldsymbol{E}=-\boldsymbol{\nabla} \Phi$ is the electric field and $\boldsymbol{D}=\varepsilon \boldsymbol{E}$ is the electric induction, where $\Phi$ is the electric potential. They satisfy

$$
\boldsymbol{\nabla} \cdot \boldsymbol{D}=-\boldsymbol{\nabla} \cdot \varepsilon(\phi) \boldsymbol{\nabla} \Phi=4 \pi \rho .
$$

The charge density $\rho(\boldsymbol{r})$ is written as

$$
\rho=e\left(Z_{1} n_{1}-Z_{2} n_{2}\right),
$$

where $e$ is the elementary charge and the two ion species have charges, $Q_{1}=Z_{1} e$ and $Q_{2}=-Z_{2} e$.

As a typical experimental geometry, our fluid system is inserted between two parallel metal plates with area $S$ and separation distance $L$ as shown in Fig. 1 . We assume $S^{1 / 2}$ $\gg L$ and neglect the effects of edge fields, for simplicity. The $z$ axis is taken perpendicularly to the plates. The surface charge densities at the upper plate at $z=L$ and the lower plate at $z=0$ are $\sigma_{L}$ and $\sigma_{0}$, respectively. The total charges on the plates are $Q_{L}=S \sigma_{L}$ and $Q_{0}=S \sigma_{0}$, respectively. The charge neutrality condition is written as

$$
Q_{L}+Q_{0}+\int d \boldsymbol{r} \rho=0,
$$

where the space integral $\int d \boldsymbol{r}(\cdots)$ is within the fluid region $(0<z<L)$. In this paper, we fix $\sigma_{L}$ and $\sigma_{0}$ and consider the electrostatic free energy dependent on inhomogeneous $\varepsilon$ and $\rho[33,36]$,

$$
F_{e}=\int d \boldsymbol{r} \frac{\varepsilon(\phi)}{8 \pi} \boldsymbol{E}^{2}=\int d \boldsymbol{r} \frac{1}{8 \pi} \boldsymbol{E} \cdot \boldsymbol{D} .
$$

On the plates at $z=0$ and $L, \Phi$ takes laterally homogeneous values $\Phi_{0}$ and $\Phi_{L}$, and

$$
D_{z}(0)=4 \pi \sigma_{0}, \quad D_{z}(L)=-4 \pi \sigma_{L} .
$$

The potential difference $\Phi_{L}-\Phi_{0}$ is a functional of $\varepsilon(\boldsymbol{r})$ and $\rho(\boldsymbol{r})$. We next change $\varepsilon(\boldsymbol{r})$ and $\rho(\boldsymbol{r})$ infinitesimally by $\delta \varepsilon(\boldsymbol{r})$ and $\delta \rho(\boldsymbol{r})$ and use the relation,

$$
\begin{aligned}
\delta\left(\varepsilon \boldsymbol{E}^{2}\right)= & -\boldsymbol{E}^{2} \delta \varepsilon+2 \boldsymbol{E} \cdot \delta \boldsymbol{D}=-\boldsymbol{E}^{2} \delta \varepsilon-2 \boldsymbol{\nabla} \cdot(\Phi \delta \boldsymbol{D}) \\
& +8 \pi \Phi \delta \rho .
\end{aligned}
$$

Because $\delta D_{z}$ should vanish on the boundaries at fixed surface charges, we find 


$$
\delta F_{e}=\int d \boldsymbol{r}\left[-\frac{1}{8 \pi} \boldsymbol{E}^{2} \delta \varepsilon+\Phi \delta \rho\right]
$$

Under Eqs. (2.2) and (2.5), the above relation yields

$$
\begin{gathered}
\frac{\delta F_{e}}{\delta \phi}=-\frac{\varepsilon_{1}}{8 \pi} \boldsymbol{E}^{2}, \\
\frac{\delta F_{e}}{\delta n_{1}}=Z_{1} e \Phi, \quad \frac{\delta F_{e}}{\delta n_{2}}=-Z_{2} e \Phi .
\end{gathered}
$$

\section{B. Solvation interaction}

In Eq. (2.1), the second term $F_{\text {ch }}$ is the chemical part,

$$
\begin{aligned}
F_{\mathrm{ch}}= & \int d \boldsymbol{r}\left\{f(\phi)+\frac{1}{2} C|\boldsymbol{\nabla} \phi|^{2}+\sum_{i=1,2}\left[k_{B} \operatorname{Tn}_{i}\left(\ln \left(v_{0} n_{i}\right)-1\right)\right.\right. \\
& \left.\left.+n_{i} \mu_{\mathrm{sol}}^{i}(\phi)\right]\right\} .
\end{aligned}
$$

The free energy density $f=f(\phi)$ is given by the Hildebrand (or Bragg-Williams) expression [31,37],

$$
\frac{v_{0} f}{k_{B} T}=\phi \ln \phi+(1-\phi) \ln (1-\phi)+\chi \phi(1-\phi),
$$

where $v_{0}$ is the molecular volume and $\chi$ is the interaction parameter dependent on the temperature $T$. The molecules of the two components have a common size

$$
a=v_{0}^{1 / 3}
$$

on the order of a few angstroms. Here the volume fraction of the ions is neglected [38]. The critical-point values of $\phi$ and $\chi$ in the absence of ions are

$$
\phi_{\mathrm{c}}=1 / 2, \quad \chi_{\mathrm{c}}=2,
$$

respectively, in the mean-field theory. That is, as $\chi \rightarrow 2,2$ $-\chi=A\left(T-T_{c}^{0}\right)$ with $A$ being a constant and $T_{c}^{0}$ being the critical temperature without ions. It is convenient to define the order parameter by

$$
\psi=\phi-1 / 2,
$$

which vanishes at the critical point without ions. The coefficient $C$ of the gradient term is of order $k_{B} T / a$ and we will use a simple form [31],

$$
C=k_{B} T \chi / a .
$$

Near the critical point, we may well use the expansion form $f=\tau \psi^{2} / 2+u \psi^{4} / 4$ [see Eq. (4.49) below].

In the second line of Eq. $(2.13), \mu_{\text {sol }}^{i}(\phi)(i=1,2)$ are the chemical potential contributions of ions due to interactions between the ions and the solvent molecules and depend on $\phi$ and $T$. In this paper, we simply assume the linear dependence,

$$
\mu_{\mathrm{sol}}^{i}(\phi)=\mu_{c}^{i}-k_{B} \operatorname{Tg}_{i} \psi \quad(i=1,2)
$$

The first terms are irrelevant constants in $F$ when the ion numbers are conserved quantities. It follows the relation
$\Delta \mu_{\alpha \beta}^{i}=k_{B} T g_{i} \Delta \phi$ with $\Delta \phi$ being the composition difference between the two phases. The linear dependence here and that in $\varepsilon(\phi)$ in Eq. (2.2) are adopted to gain the physical consequences in the simplest manner and should not be taken too seriously. We then have the bilinear solvation interaction $\left(\propto \psi n_{i}\right)$ in $F$ among the ions and the composition fluctuations $[32,39]$. In aqueous solutions, the coupling constants $g_{i}$ are positive for hydrophilic ions and can much exceed unity for small ions such as $\mathrm{Li}^{+}$or $\mathrm{Na}^{+}$, while they should be negative for hydrophobic organic ions [14]. They should be larger for small multivalent ions such as $\mathrm{Mg}^{2+}$ or $\mathrm{Al}^{3+}$. As will be summarized in Appendix A, we recently examined the effect of this solvation interaction in near-critical one-phase states [32]. In this work, its effect will be examined in two-phase states. In addition, $1 / \varepsilon(\phi)$ in the Born formula Eq. (1.1) may be expanded in powers of $\psi$ near the critical point to give $[33,40]$

$$
\left(g_{i}\right)_{\mathrm{Born}}=Z_{i}^{2} e^{2} \varepsilon_{1} / 2 k_{B} T \varepsilon_{c}^{2} R_{\text {ion }}^{i},
$$

where $\varepsilon_{c}$ is the dielectric constant at the critical composition and $R_{\text {ion }}^{i}$ is the ion radius of the species $i$. However, because of the defects of the Born theory, we will treat $g_{i}$ as free parameters not using Eq. (2.20).

Hydrophilic ions and several water molecules (those of the more polar component in a mixture) form a complex structure and its effective radius $R_{\text {shell }}^{i}$, called the solvation (hydration) shell radius, can be a few angstroms and is larger than the bare ion radius $R_{\text {ion }}^{i}[3,4]$. In a number of experiments, water molecules have been extracted together with hydrophilic ions in a less polar phase with a small water concentration. In nitrobenzene (NB) -water at room temperatures, the number of coextracted water molecules in a NBrich phase was estimated to be 4 for $\mathrm{Na}^{+}, 6$ for $\mathrm{Li}^{+}$, and 15 for $\mathrm{Ca}^{2+}[14]$. For liquid-vapor systems, where ions are absent in vapor, Levin and Flores-Mena [27] argued that ions in liquid cannot approach the interface within the distance of $R_{\text {shell }}^{i}$ in the thin interface limit $\xi \rightarrow 0$. In our theory, $R_{\text {shell }}^{i}$ does not appear explicitly, but ions in the more polar region are strongly repelled from the interface outside the distance of $\xi$ for large $g_{i}$. Therefore, our present treatment is justified in the case $\xi \gtrsim R_{\text {shell }}^{i}$.

\section{Image interaction}

As will be discussed in Appendix B, the last term $F_{\text {im }}$ arises from a deformation of the self-interaction of ions due to inhomogeneous $\varepsilon$. It originates from the discrete nature of ions, while the electric field $\Phi$ in Eq. (2.4) is produced by the smoothly coarse-grained charge density $\rho$. If an ion is close to an interface, the chemical potential contribution $\mu_{\mathrm{im}}^{i}$ $=\delta F_{\mathrm{im}} / \delta n_{i}$ is the so-called image potential in the previous papers [24-26]. This interaction can be important close to an interface far below the critical point or a boundary wall.

In this paper, assuming weak or moderate spatial variations of $\varepsilon$, we derive the following integral form,

$$
F_{\mathrm{im}}=\iint d \boldsymbol{r} d \boldsymbol{r}^{\prime} m(\boldsymbol{r}) \boldsymbol{\nabla}^{\prime} I\left(\boldsymbol{r}, \boldsymbol{r}^{\prime}\right) \cdot \nabla^{\prime} \phi\left(\boldsymbol{r}^{\prime}\right),
$$

where $\nabla^{\prime}=\partial / \partial \boldsymbol{r}^{\prime}$. We define a new variable, 


$$
m=Z_{1}^{2} n_{1}+Z_{2}^{2} n_{2},
$$

which coincides with the total ion density,

$$
n=n_{1}+n_{2} \text {, }
$$

in the monovalent case $Z_{1}=Z_{2}=1$. If the boundary effects are neglected, the function $I\left(\boldsymbol{r}, \boldsymbol{r}^{\prime}\right)$ depends only on the difference $\boldsymbol{r}-\boldsymbol{r}^{\prime}$ as

$$
I\left(\boldsymbol{r}, \boldsymbol{r}^{\prime}\right)=\frac{B_{0}}{\left|\boldsymbol{r}-\boldsymbol{r}^{\prime}\right|^{2}} e^{-2 \kappa\left|\boldsymbol{r}-\boldsymbol{r}^{\prime}\right|} .
$$

Near the critical point, the coefficient $B_{0}$ is given by

$$
B_{0}=e^{2} \varepsilon_{1} / 8 \pi \varepsilon_{c}^{2},
$$

where $\varepsilon_{c}=\varepsilon_{0}+\varepsilon_{1} / 2$ is the dielectric constant at the critical point and $\kappa$ is the Debye-Hückel wave number. When the ion densities vary significantly in space in a phase-separated state, $\kappa$ may be taken as the space-dependent local value [see sentences below Eq. (3.8)] [24] or the value in the more polar phase [25].

In our numerical analysis, we will set $\varepsilon_{1}=4 \varepsilon_{0} / 3$ or $\varepsilon_{1}$ $=4 \varepsilon_{c} / 5$ for $\phi_{c}=1 / 2$. Then $\left|\varepsilon_{1} \psi\right| / \varepsilon_{c}$ is at most 0.4 in strong segregation of composition. Our one-dimensional form of $F_{\text {im }}$ to be presented in Eq. (3.7) below overestimates the usual expression in the case $\varepsilon_{1} \gg \varepsilon_{0}$ [25] by a factor of 2 in strong segregation. See Appendix B.

\section{Chemical potentials and equilibrium}

We calculate the chemical potentials $\mu_{1}=\delta F / \delta n_{1}$ and $\mu_{2}$ $=\delta F / \delta n_{2}$ of the ions and $h=\delta F / \delta \phi$. Here $h=\left(\mu_{\mathrm{A}}-\mu_{\mathrm{B}}\right) / v_{0}$ in terms of the chemical potentials $\mu_{\mathrm{A}}$ and $\mu_{\mathrm{B}}$ of the two components of the mixture [37]. Using Eqs. (2.11) and (2.12), we obtain

$$
\begin{aligned}
& \mu_{1}=k_{B} T \ln \left(v_{0} n_{1}\right)+Z_{1} e \Phi+\mu_{\mathrm{sol}}^{1}+\mu_{\mathrm{im}}^{1}, \\
& \mu_{2}=k_{B} T \ln \left(v_{0} n_{2}\right)-Z_{2} e \Phi+\mu_{\mathrm{sol}}^{2}+\mu_{\mathrm{im}}^{2} .
\end{aligned}
$$

From Eqs. (2.11), (2.13), and (2.19), we find

$$
h=f^{\prime}(\phi)-C \nabla^{2} \phi-\frac{\varepsilon_{1}}{8 \pi} \boldsymbol{E}^{2}-k_{B} T \sum_{i=1,2} g_{i} n_{i}+h_{\mathrm{im}},
$$

where $f^{\prime}=\partial f / \partial \phi$. In these expressions, the last two terms arise from the solvation and image interactions, respectively, and we define

$$
\mu_{\mathrm{im}}^{i}=\frac{\delta F_{\mathrm{im}}}{\delta n_{i}}, \quad h_{\mathrm{im}}=\frac{\delta F_{\mathrm{im}}}{\delta \phi} .
$$

If Eq. (2.24) is used with a constant $\kappa$, we have

$$
\begin{gathered}
\mu_{\mathrm{im}}(\boldsymbol{r}) \equiv \mu_{\mathrm{im}}^{i} / Z_{i}^{2}=\int d \boldsymbol{r}^{\prime} \nabla^{\prime} I\left(\boldsymbol{r}, \boldsymbol{r}^{\prime}\right) \cdot \nabla^{\prime} \psi\left(\boldsymbol{r}^{\prime}\right), \\
h_{\mathrm{im}}(\boldsymbol{r})=\int d \boldsymbol{r}^{\prime} \nabla^{\prime} I\left(\boldsymbol{r}, \boldsymbol{r}^{\prime}\right) \cdot \nabla^{\prime} m\left(\boldsymbol{r}^{\prime}\right) .
\end{gathered}
$$

In our numerical analysis, we have used these expressions in Eqs. (2.26) and (2.27) for simplicity [see Eq. (3.7)].
In equilibrium, $\mu_{1}, \mu_{2}$, and $h$ are constants in space. The ion distributions are written in terms of the potential $\Phi$ and the order parameter $\psi=\phi-1 / 2$ as

$$
\begin{aligned}
& n_{1}=n_{1}^{0} \exp \left[g_{1} \psi-\frac{Z_{1}}{k_{\mathrm{B}} T}\left(e \Phi+Z_{1} \mu_{\mathrm{im}}\right)\right], \\
& n_{2}=n_{2}^{0} \exp \left[g_{2} \psi+\frac{Z_{2}}{k_{B} T}\left(e \Phi-Z_{2} \mu_{\mathrm{im}}\right)\right],
\end{aligned}
$$

where we have used Eq. (2.19) for $\mu_{\text {sol }}^{i}$ and Eq. (2.29) for $\mu_{\mathrm{im}}^{i}$. The coefficients $n_{1}^{0}$ and $n_{2}^{0}$ are determined from the conditions $N_{1}=\int d \boldsymbol{r} n_{1}=$ const. and $N_{2}=\int d \boldsymbol{r} n_{2}=$ const.

\section{NUMERICAL METHOD AND ONE-DIMENSIONAL PROFILES WITH BOUNDARIES}

We consider one-dimensional equilibrium situations in which all the quantities in $F$ depend only on $z$. We introduce normalized ion densities by

$$
c_{1}(z)=v_{0} n_{1}(z), \quad c_{2}(z)=v_{0} n_{2}(z) .
$$

As typical values, if $n_{1}=10^{-4} \mathrm{~mole} / \mathrm{cm}^{3}$ and $a=3.1 \AA$, we obtain $c_{1}=1.8 \times 10^{-3}$. We simply call $c_{1}$ and $c_{2}$ as concentrations, supposing $R_{\text {shell }}^{i} \sim a$. We also introduce a dimensionless electric potential,

$$
U(z)=e \Phi(z) / k_{B} T
$$

In Appendix $\mathrm{C}$, we will write down the equilibrium equations for $\psi=\phi-1 / 2, c_{1}, c_{2}$, and $U$. There appears a parameter $A$ representing the strength of the charges,

$$
A=\pi e^{2} / 4 a \varepsilon_{c} k_{B} T=\pi \ell_{\mathrm{B} c} / 4 a,
$$

where $a=v_{0}^{1 / 3}$ is the molecular size and

$$
\ell_{\mathrm{B} c}=e^{2} / \varepsilon_{c} k_{B} T
$$

is the Bjerrum length at the critical dielectric constant.

Our numerical calculations are all in the monovalent case $Z_{1}=Z_{2}=1$. We integrated the time-dependent equations,

$$
\begin{aligned}
& \frac{\partial \phi}{\partial t}=-\frac{\delta F}{\delta \phi}+\left\langle\frac{\delta F}{\delta \phi}\right\rangle, \\
& \frac{\partial U}{\partial t}=\nabla \cdot \varepsilon \nabla U+4 \pi \rho,
\end{aligned}
$$

until steady solutions were nearly reached, where $\langle\cdots\rangle$ is the space average. Notice that $h$ in Eq. (2.27) becomes equal to the space average $\langle\delta F / \delta \phi\rangle$ in equilibrium. Thus the steady solutions of Eqs. (3.5) and (3.6) are the equilibrium solutions. We used $h_{\text {im }}$ in Eq. (2.29) as $\delta F_{\text {im }} / \delta \phi$ in $\delta F / \delta \phi$ and $n_{1}$ and $n_{2}$ in Eq. (2.30) to calculate $m=n$ and $\rho$. These equations do not represent the real dynamics, but conveniently give the equilibrium solutions.

In $\mu_{\text {im }}(z)$ and $h_{\text {im }}(z)$ in Eq. (2.29) we replace $\exp (-2 \kappa \mid \boldsymbol{r}$ $\left.-\boldsymbol{r}^{\prime} \mid\right)$ in $I\left(\boldsymbol{r}, \boldsymbol{r}^{\prime}\right)$ by $\exp \left(-2 \kappa\left|z-z^{\prime}\right|\right)$ for simplicity and integrate over $x^{\prime}$ and $y^{\prime}$ to obtain 


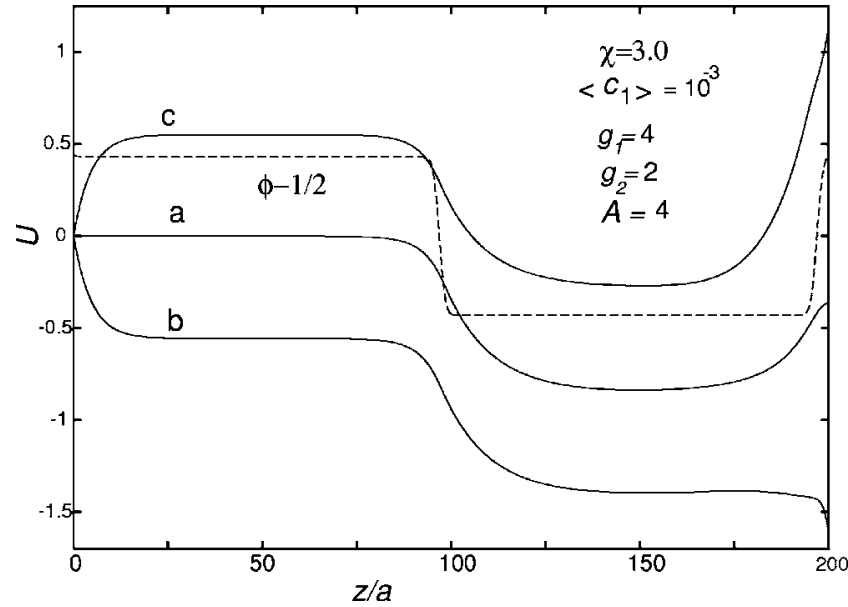

FIG. 2. Normalized electric potential $U(z)=e \Phi(z) / k_{B} T$ between parallel plates with $L=200 a$, where we set $\Phi(0)=0$. The surface charge $\sigma_{0}\left(=-\sigma_{L}\right)$ is $0(\mathrm{a}), 2.5 \times 10^{-3} e / a^{2}(\mathrm{~b})$, and $-2.5 \times 10^{-3} e / a^{2}$ (c). The curve of $\psi(z)=\phi(z)-1 / 2$ (broken line) indicates the presence of an interface in the middle and a wetting layer in contact with the wall at $z=L$.

$$
\mu_{\text {im }}(z)=2 \pi B_{0} \int \frac{d z^{\prime}}{z-z^{\prime}} e^{-2 \kappa\left|z-z^{\prime}\right|} \frac{d}{d z^{\prime}} \psi\left(z^{\prime}\right),
$$

where $B_{0}$ is given in Eq. (2.25). See Eq. (B9). The $h_{\mathrm{im}}(z)$ is obtained if $\psi\left(z^{\prime}\right)$ is replaced by $m\left(z^{\prime}\right)$ in Eq. (3.7). We neglected the image interaction in the regions $z<20 a$ and $L$ $-z<20 a$ close to the boundaries. As the Debye-Hückel wave number in Eq. (3.7), we used its local value in the monovalent case,

$$
\kappa(z)=\left[4 \pi n(z) e^{2} / \varepsilon(\phi(z)) k_{B} T\right]^{1 / 2} .
$$

We also performed numerical analysis by setting $\kappa$ equal to the bulk value $\kappa_{\alpha}$ in Eq. (4.2). A noticeable difference can be seen only in the long-distance behavior of the electric potential $\Phi$ in the phase $\beta$ at very small ion densities (see Fig. 13 below). The calculated values of the excess surface tension are different between these two choices only by a few percents.

In Fig. 2, we show numerically calculated profiles of the normalized electric potential $U(z)$, for $\chi=3, g_{1}=4, g_{2}=2$, and $A=4$ in the geometry of Fig. 1 with $L=200 a$. The space averages are $\langle\psi\rangle=0$ and $\left\langle c_{1}\right\rangle=\left\langle c_{2}\right\rangle=0.001$. The three curves correspond to the surface charge densities given by $\sigma_{0}=$ $-\sigma_{L}=0$ (a), $2.5 \times 10^{-3} e / a^{2}(\mathrm{~b})$, and $-2.5 \times 10^{-3} e / a^{2}$ (c). Note that $D_{z}=-\varepsilon d \Phi / d z$ satisfies the boundary condition in Eq. (2.8). On the composition deviation $\psi=\phi-1 / 2$, we impose the boundary condition [41],

$$
\psi= \pm(2 \sqrt{2})^{-1} a \psi^{\prime}+0.5
$$

where $\psi^{\prime}=d \psi / d z,+$ is for $z=0$, and - is for $z=L$. Under Eq. (3.9), the boundary walls are wetted by the more polar phase. The electric potential exhibits a jump near the interface, whose spatial scale is of the order of the screening length $1 / \kappa$ longer than the interface thickness $\xi$ in the present case. This suggests the presence of an electric double layer at the interface, as will be discussed in Sec. IV B. The surface charges $\sigma_{0}$ and $\sigma_{L}$ are screened by a fraction of the ions in the fluid.

\section{PLANAR INTERFACE}

From Fig. 2, we can see that the interface is not affected by the surface charges and the wetting layer as long as the distances to the boundaries are much longer than the screening length. We focus our attention to a planar interface separating two phases $\alpha$ and $\beta$. The volume fraction $\phi_{\alpha}$ in the phase $\alpha$ is larger than that $\phi_{\beta}$ in the phase $\beta$, so the dielectric constant is larger in the phase $\alpha$ than in the phase $\beta$,

$$
\varepsilon_{\alpha}=\varepsilon_{c}+\varepsilon_{1} \psi_{\alpha}>\varepsilon_{\beta}=\varepsilon_{c}+\varepsilon_{1} \psi_{\beta} .
$$

The interface structure is determined by the six dimensionless parameters, $\chi, g_{1}, g_{2}, \epsilon_{1} / \epsilon_{c}, A$, and the bulk ion concentration $c_{1 \alpha}$ in the phase $\alpha$. In this paper, we set $\epsilon_{1} / \epsilon_{c}=0.8$, but the other parameters are varied.

\section{A. General relations}

We assume that there are significant ion densities in the two phases such that the bulk screening lengths $1 / \kappa_{\alpha}$ and $1 / \kappa_{\beta}$ in the two phases are shorter than the cell length $L$. Here,

$$
\begin{aligned}
\kappa_{K}^{2} & =4 \pi e^{2}\left(Z_{1}^{2} n_{1 K}+Z_{2}^{2} n_{2 K}\right) / \varepsilon_{K} k_{B} T \\
& =16 A\left(Z_{1}^{2} c_{1 K}+Z_{2}^{2} c_{2 K}\right) / \hat{\varepsilon}_{K} a^{2},
\end{aligned}
$$

where $A$ is defined by Eq. (3.3) and $\hat{\varepsilon}_{K}=\varepsilon_{K} / \varepsilon_{c}$. Hereafter, the subscript $K$ stands for $\alpha$ or $\beta$. The ion densities in the two bulk phases are written as

$$
n_{1 K}=v_{0}^{-1} c_{1 K}, \quad n_{2 K}=v_{0}^{-1} c_{2 K} .
$$

Far from the interface, the charge density $\rho(z)$ tends to zero and the electric potential $\Phi(z)$ tends to constants, $\Phi_{\alpha}$ and $\Phi_{\beta}$. The charge neutrality conditions far from the interface in the two bulk phases are written as

$$
Z_{1} n_{1 K}=Z_{2} n_{2 K}
$$

Since $\mu_{\text {im }}^{i}$ vanish far from the interface, the ion densities in the two bulk phases are written as

$$
\begin{gathered}
n_{1 K}=n_{1}^{0} \exp \left[-\left(Z_{1} e \Phi_{K}+\mu_{\mathrm{sol}}^{1 K}\right) / k_{B} T\right], \\
n_{2 K}=n_{2}^{0} \exp \left[\left(Z_{2} e \Phi_{K}-\mu_{\mathrm{sol}}^{2 K}\right) / k_{B} T\right] .
\end{gathered}
$$

We define the solvation chemical potentials in the bulk,

$$
\mu_{\mathrm{sol}}^{1 K}=\mu_{\mathrm{sol}}^{1}\left(\phi_{K}\right), \quad \mu_{\mathrm{sol}}^{2 K}=\mu_{\mathrm{sol}}^{2}\left(\phi_{K}\right) .
$$

The charge neutrality condition (4.4) yields

$$
\begin{aligned}
\Phi_{\alpha}-\Phi_{\beta} & =\frac{1}{\left(Z_{1}+Z_{2}\right) e}\left[\Delta \mu_{\alpha \beta}^{2}-\Delta \mu_{\alpha \beta}^{1}\right] \\
& =\frac{k_{B} T}{\left(Z_{1}+Z_{2}\right) e}\left(g_{1}-g_{2}\right) \Delta \phi .
\end{aligned}
$$

Use has been made of the definition (1.2). The composition difference is written as 


$$
\Delta \phi=\phi_{\alpha}-\phi_{\beta}>0
$$

The first line in Eq. (4.8) is the general expression for the potential difference [15-19], while the second line is a simplified expression under the assumption (2.19). This potential difference depends on the ion density only through $\phi_{\alpha}$ and $\phi_{\beta}$ [see Eq. (4.38) below]. Notice that the potential difference arises in the asymmetric case $\left(g_{1} \neq g_{2}\right)$ only and vanishes in the symmetric case $\left(g_{1}=g_{2}\right)$. More strongly in our model, we find $\Phi(z)=\rho(z)=0$ even around the interface for $g_{1}=g_{2}$.

Furthermore, the charge densities in the phase $\beta$ are reduced as

$$
\frac{n_{1 \beta}}{n_{1 \alpha}}=\frac{n_{2 \beta}}{n_{2 \alpha}}=\exp \left(-\frac{\epsilon_{r}}{k_{B} T}\right) .
$$

The degree of the ion-density reduction is represented by the energy $\epsilon_{r}$ written as

$$
\begin{aligned}
\epsilon_{r} & =\frac{Z_{2}}{Z_{1}+Z_{2}} \Delta \mu_{\alpha \beta}^{1}+\frac{Z_{1}}{Z_{1}+Z_{2}} \Delta \mu_{\alpha \beta}^{2} \\
& =\frac{k_{B} T}{Z_{1}+Z_{2}}\left(Z_{2} g_{1}+Z_{1} g_{2}\right) \Delta \phi .
\end{aligned}
$$

The second line holds under Eq. (2.19) as in the second line of Eq. (4.8). For example, if $\Delta \mu_{\alpha \beta}^{1} / k_{B} T=\Delta \mu_{\alpha \beta}^{2} / k_{B} T=10$ and 60 , the ion reduction factor becomes $e^{-10}=2.4 \times 10^{-4}$ and $e^{-60}=0.67 \times 10^{-26}$, respectively, in the monovalent case. In the latter case, the ion density is virtually zero in the phase $\beta$. The Debye-Hückel wave numbers in Eq. (4.2) satisfy

$$
\kappa_{\beta} / \kappa_{\alpha}=\left(\varepsilon_{\alpha} / \varepsilon_{\beta}\right)^{1 / 2} \exp \left(-\epsilon_{r} / 2 k_{B} T\right) .
$$

If $\epsilon_{r} / k_{B} T$ is considerably larger than unity, we have $n_{1 \beta}$ $\ll n_{1 \alpha}, n_{2 \beta} \ll n_{2 \alpha}$, and $1 / \kappa_{\beta} \gg 1 / \kappa_{\alpha}$. On the other hand, as we approach the critical point, $\Delta \phi$ and $\epsilon_{\mathrm{r}} / k_{B} T$ eventually become small, where the heterogeneity in the ion distributions is weak. If $g_{1}$ and $g_{2}$ are large, this crossover occurs very close to the critical point.

We show numerical results in the monovalent case. In Figs. 3-5, we set $\chi=2.3, g_{1}=4, g_{2}=2, A=4$, and $\langle\psi\rangle=0$ with $L=100 a$. At the boundaries at $z=0$ and $L$, we impose the conditions,

$$
\psi^{\prime}=0, \quad \Phi^{\prime}=0 .
$$

If $\kappa_{\beta} L \gg 1$, we may realize the interface profiles not affected by the boundary effects. Figure 3 displays the ion densities and the charge density for $c_{1 \alpha}=1.75 \times 10^{-3}$. The charge density changes rather abruptly between the maximum and the minimum on the scale of $\xi$ [42], but slowly tends to zero outside the interval of them on the scale of $1 / \kappa_{\alpha}$ or $1 / \kappa_{\beta}$. We can also see a rounded peak in $c_{1}(z)$ in the phase $\alpha$. The latter is due to the factor $\exp (-U)$ where $U=e \Phi / k_{B} T$ decreases by 0.2 on the left of the interface. This peak tends to disappear for larger $g_{i}$. For three values of $c_{1 \alpha}$, Figs. 4 and 5 illustrate the electric potential $\Phi(z)$ and the composition deviation $\psi(z)$, respectively. They are consistent with Eq. (4.8). For example, for the case (b) with $c_{1 \alpha}=0.018$ in Figs. 4 and 5, we numerically obtain $\psi_{\alpha}=0.3374, \quad \psi_{\beta}=-0.3137$, and $\Delta U$

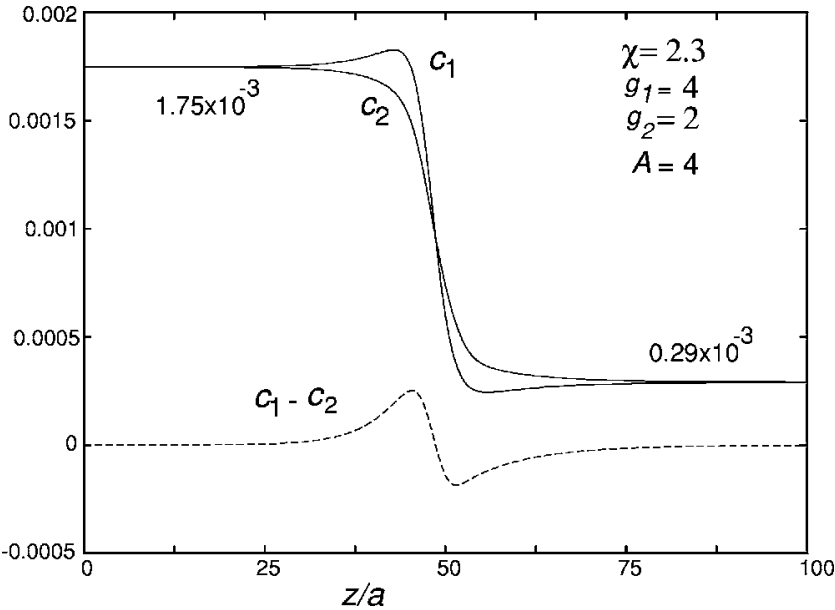

FIG. 3. Normalized ion densities $c_{1}(z)$ and $c_{2}(z)$ around an interface where $c_{1 \alpha}=c_{2 \alpha}=1.75 \times 10^{-3}$ and $c_{1 \beta}=c_{2 \beta}=0.29 \times 10^{-3}$.

$=e\left(\Phi_{\alpha}-\Phi_{\beta}\right) / k_{B} T=0.6410$ to confirm the coincidence of the left-hand and right-hand sides of Eq. (4.8). Furthermore, the ion reduction factor in Eq. (4.10) is $\exp [-3 \Delta \phi]=0.146$ for this case, which is consistent with the numerical value $c_{1 \beta}$ $=0.00264$.

Near a water-air interface without ions in air, the microscopic calculations [6] showed a difference between the cation and anion distributions, which decreases in the order of the series: $\mathrm{NaI}<\mathrm{NaBr}<\mathrm{NaCl}<\mathrm{NaF}$ in agreement with the order of the depletion-layer-thickness $\lambda_{s}$ in Eq. (1.3). The distributions are most different for $\mathrm{NaI}$ and almost coincide for NAF. This suggests that the hydration chemical potentials are most different for $\mathrm{Na}^{+}$and $\mathrm{I}^{-}$and are nearly equal for $\mathrm{Na}^{+}$and $\mathrm{F}^{-}$. See the sentences around Eq. (4.20).

\section{B. Charge density and electric potential}

Emergence of a charge density around an interface gives rise to a change of the electric potential across the interface. To show this in the case $\kappa_{\beta} L \gg 1$, we integrate Eq. (2.4) to obtain the electric induction,

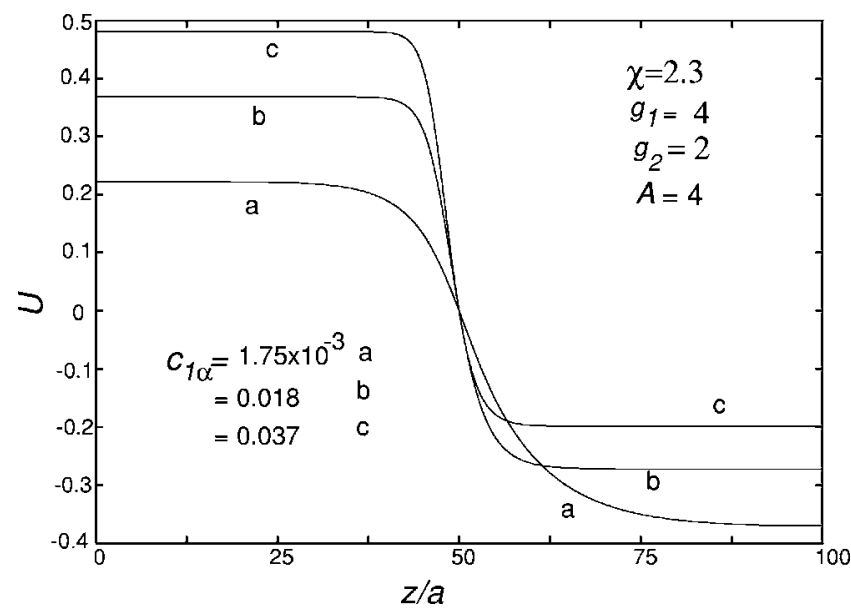

FIG. 4. Normalized electric potential $U(z)=e \Phi(z) / k_{B} T$ for three cases of $c_{1 \alpha}=1.75 \times 10^{-3}, 0.018$, and 0.037 , where we set $\Phi(50 a)$ $=0$. 


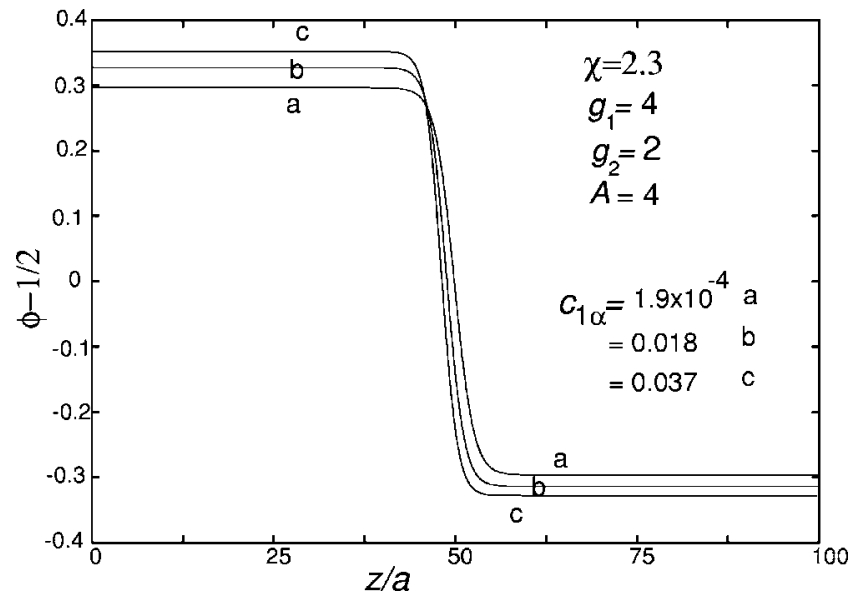

FIG. 5. Order parameter profile $\psi(z)=\phi(z)-1 / 2$ for three cases of $c_{1 \alpha}=1.9 \times 10^{-4}$ (a), $0.018(\mathrm{~b})$, and 0.037 (c). For the smallest $c_{1 \alpha}$, $\psi(z)$ is almost equal to the profile without ions.

$$
D(z)=-\varepsilon(z) \Phi^{\prime}(z)=4 \pi \int_{-\infty}^{z} d z^{\prime} \rho\left(z^{\prime}\right)
$$

where the lower bound is pushed to $-\infty$. The $z$ dependence of $\varepsilon$ stems from that of $\phi$. To ensure $D(\infty)=0$, we need to require the total charge neutrality condition $\int_{-\infty}^{\infty} d z \rho(z)=0$. Dividing Eq. (4.14) by $\varepsilon$ and integrating it, we obtain

$$
\Phi(z)=\Phi_{\alpha}-4 \pi \int_{-\infty}^{z} d z^{\prime} \rho\left(z^{\prime}\right)\left[\zeta(z)-\zeta\left(z^{\prime}\right)\right],
$$

where we introduce

$$
\zeta(z)=\int_{z_{\mathrm{int}}}^{z} d z^{\prime} \frac{1}{\varepsilon\left(z^{\prime}\right)} .
$$

The right-hand side of Eq. (4.15) is independent of the lower bound $z_{\text {int }}$ in Eq. (4.16). However, it is convenient to choose $z_{\text {int }}$ as the interface position in the Gibbs construction [43] [see Eq. (4.42) below]; then, $\zeta \cong\left(z-z_{\text {int }}\right) / \varepsilon_{K}$ far from the interface where $K=\alpha$ or $\beta$ in the $\alpha$ or $\beta$ region. Here $z \int_{-\infty}^{z} d z^{\prime} \rho\left(z^{\prime}\right) \propto z \Phi^{\prime}(z)$ should tend to zero for large $z$ [since $\rho(z) \propto e^{-\kappa_{\beta} z}$ as $z \rightarrow \infty$ ], we have

$$
\Phi_{\alpha}-\Phi_{\beta}=-4 \pi \int_{-\infty}^{\infty} d z^{\prime} \rho\left(z^{\prime}\right) \zeta\left(z^{\prime}\right),
$$

where the upper bound is also pushed to infinity. We are interested in the potential value at the interface position,

$$
\Phi_{\alpha}-\Phi\left(z_{\mathrm{int}}\right)=-4 \pi \int_{-\infty}^{z_{\mathrm{int}}} d z^{\prime} \rho\left(z^{\prime}\right) \zeta\left(z^{\prime}\right) .
$$

As can be inferred from the profile of $c_{1}-c_{2}=a^{3} \rho / e$ in Fig. 3 , $\Phi$ should decrease from the maximum to the minimum point of $\rho$ by a small amount $(\Delta \Phi)_{\text {sol }} \propto n_{1 \alpha}$ [42]. For $g_{1}$ $>g_{2} \gg 1$ with $\Delta \phi \sim 1$, it is estimated as

$$
(\Delta \Phi)_{\mathrm{sol}} \sim e n_{1 \alpha} \xi^{2} \ln \left(g_{1} / g_{2}\right) / \varepsilon_{\alpha}
$$

If $\xi \sim a$, we obtain $e(\Delta \Phi)_{\mathrm{sol}} / k_{B} T \sim c_{1 \alpha} A \ln \left(g_{1} / g_{2}\right)$.
In view of the inequality $|\rho(z)|<2 e Z_{1} n_{1 \alpha}$, it is surprising that $\Phi_{\alpha}-\Phi_{\beta}$ in Eq. (4.8) remains nonvanishing even in the dilute limit of ions. In the low density limit of the ions, therefore, $\rho$ should change over the screening length outside the interval between the extremum points of $\rho$. Furthermore, if $\kappa_{\beta} \ll \kappa_{\alpha}$, the typical variation of $\Phi$ in the $\alpha$ region should be smaller than that in the $\beta$ region by the factor $\varepsilon_{\beta} \kappa_{\beta} / \varepsilon_{\alpha} \kappa_{\alpha}$ owing to the continuity of $D(z)$ in Eq. (4.14). In the case $\kappa_{\beta} \ll \kappa_{\alpha} \ll \xi^{-1}$, the potential decrease Eq. (4.18) behaves as

$$
\Phi_{\alpha}-\Phi\left(z_{\text {int }}\right) \cong(\Delta \Phi)_{\mathrm{sol}}+2 \frac{\varepsilon_{\beta} \kappa_{\beta}}{\varepsilon_{\alpha} \kappa_{\alpha}} \frac{k_{B} T}{e} \sinh \left(\frac{\Delta U}{2}\right),
$$

where $\Delta U=e\left(\Phi_{\alpha}-\Phi_{\beta}\right) / k_{B} T$. See discussions below Eq. (4.56). The electric field on the left-hand side of the maximum point of $\rho$ in the $\alpha$ region is negligible in the limit $n_{\beta}$ $\rightarrow 0$. In accord with this result, $\rho=\Phi=0$ was assumed in the water region in the previous papers [24-27].

\section{Grand potential density}

We consider the grand potential density in onedimensional equilibrium situations,

$$
\begin{aligned}
\omega(z)= & f+\frac{C}{2}\left|\phi^{\prime}\right|^{2}-h \phi+\frac{\varepsilon}{8 \pi} E^{2}+m \mu_{\mathrm{im}} \\
& +\sum_{i=1,2} n_{i}\left[k_{B} T\left(\ln \left(v_{0} n_{i}\right)-1\right)+\mu_{\mathrm{sol}}^{i}(\phi)-\mu_{i}\right],
\end{aligned}
$$

where $\mu_{\text {im }}$ is defined by Eq. (2.29) and $h, \mu_{1}$, and $\mu_{2}$ are constants (Lagrange multipliers). The grand potential is given by

$$
\Omega=\int d \boldsymbol{r} \omega=F-\int d \boldsymbol{r}\left[h \boldsymbol{\phi}+\sum_{i=1,2} \mu_{i} n_{i}\right],
$$

where $F$ is the Helmholtz free energy (2.1). If we minimize $\Omega$ as a functional of $\phi, n_{1}$, and $n_{2}$, we are led to Eqs. (2.26) and (2.27) with Eq. (2.28). To obtain an interface, we impose the boundary condition $\phi \rightarrow \phi_{K}(K=\alpha, \beta)$ with $\phi_{\alpha}>\phi_{\beta}$ far from the interface.

For the interface solution, we can prove the relation,

$$
\frac{d}{d z}\left[\omega-C\left|\phi^{\prime}\right|^{2}-m \mu_{\mathrm{im}}\right]=-n_{1}^{\prime} \mu_{\mathrm{im}}^{1}-n_{2}^{\prime} \mu_{\mathrm{im}}^{2}-\phi^{\prime} h_{\mathrm{im}},
$$

where the prime denotes taking the derivative $d / d z$. We may see that the $z$ integration of the right-hand side of Eq. (4.23) vanishes. In fact, if we shift $z$ in $\phi(z), n_{i}(z)$, and $\Phi(z)$ to $z$ $+\delta \zeta$, the incremental change of $F_{\text {im }}$ is

$$
\delta F_{\mathrm{im}}=\int d \boldsymbol{r}\left[n_{1}^{\prime} \mu_{\mathrm{im}}^{1}+n_{2}^{\prime} \mu_{\mathrm{im}}^{2}+\phi^{\prime} h_{\mathrm{im}}\right] \delta \zeta .
$$

For constant $\delta \zeta$, we have $\delta F_{\text {im }}=0$ from the translational invariance of the interface position. This is justified if the interface is located very far from the boundary walls. Then 
integration of Eq. (4.23) indicates coincidence of the bulk values of $\omega(z)$

$$
\begin{aligned}
\omega_{\infty} & =f\left(\phi_{\alpha}\right)-h \phi_{\alpha}-k_{B} T n_{\alpha} \\
& =f\left(\phi_{\beta}\right)-h \phi_{\beta}-k_{B} T n_{\beta} .
\end{aligned}
$$

Here, $n_{\alpha}$ and $n_{\beta}$ are the bulk values of $n=n_{1}+n_{2}$. We have eliminated $\mu_{1}$ and $\mu_{2}$ with the aid of $\sum_{i} n_{i}\left[\mu_{i}-k_{B} T \ln n_{i}\right.$ $\left.-\mu_{\mathrm{sol}}^{i}\right] \rightarrow 0$ far from the interface which follows from Eqs. (2.26) and (4.4). The homogeneity of $h$ in Eq. (2.27) yields

$$
\begin{aligned}
h & =f^{\prime}\left(\phi_{\alpha}\right)-k_{B} T\left(g_{1} n_{1 \alpha}+g_{2} n_{2 \alpha}\right) \\
& =f^{\prime}\left(\phi_{\beta}\right)-k_{B} T\left(g_{1} n_{1 \beta}+g_{2} n_{2 \beta}\right) .
\end{aligned}
$$

Now Eqs. (4.25) and (4.26) supplemented with Eqs. (2.14) and (4.10) constitute a closed set of equations that determine the equilibrium compositions $\phi_{\alpha}$ and $\phi_{\beta}$, as will be examined in Sec. IV G.

\section{Surface tension}

In terms of the grand potential density in Eq. (4.21), the surface tension is written as

$$
\gamma=\int d z\left[\omega(z)-\omega_{\infty}\right]
$$

where the integrand tends to zero away from the interface owing to Eq. (4.25). We eliminate $\mu_{1}$ and $\mu_{2}$ in $\omega(z)$ using Eq. (2.29) to have a simpler expression,

$$
\omega(z)=f+\frac{C}{2}\left|\phi^{\prime}\right|^{2}-h \phi-k_{B} T n+\frac{\varepsilon}{8 \pi} E^{2}-\rho \Phi .
$$

Use of Eq. (2.4) gives

$$
\gamma=\int d z\left[f(\phi)+\frac{C}{2}\left|\phi^{\prime}\right|^{2}-h \phi-k_{B} T n-\frac{\rho}{2} \Phi-\omega_{\infty}\right] .
$$

On the other hand, the surface tension without ions is expressed as

$$
\gamma_{0}=\int d z\left[f\left(\phi_{0}\right)+\frac{1}{2} C\left|\phi_{0}^{\prime}\right|^{2}-f_{0}\right],
$$

where $\phi_{0}(z)$ is the interface solution without ions, tending to $\phi_{0 \alpha}$ and $\phi_{0 \beta}$ as $z \rightarrow \mp \infty$, respectively. It satisfies [37]

$$
\begin{gathered}
f^{\prime}\left(\phi_{0}\right)-C \phi_{0}^{\prime \prime}=0 \\
f\left(\phi_{0}\right)-\frac{1}{2} C\left|\phi_{0}^{\prime}\right|^{2}=f_{0}=\text { const. }
\end{gathered}
$$

at any $z$ so that $f_{0}=f\left(\phi_{0 \alpha}\right)=f\left(\phi_{0 \beta}\right)$. For the special form of $f$ in Eq. (2.14), we have $h=0$ (in the zeroth order) and $\phi_{0 \alpha}$ $+\phi_{0 \beta}=1[48]$.

Let us consider the excess surface tension,

$$
\Delta \gamma=\gamma-\gamma_{0} .
$$

In Fig. 6, we show the normalized excess surface tension $a^{2} \Delta \gamma / k_{B} T$ versus $c_{1 \alpha}$ at $A=4$ for $\chi=3,2.3$, and 2.05 , for

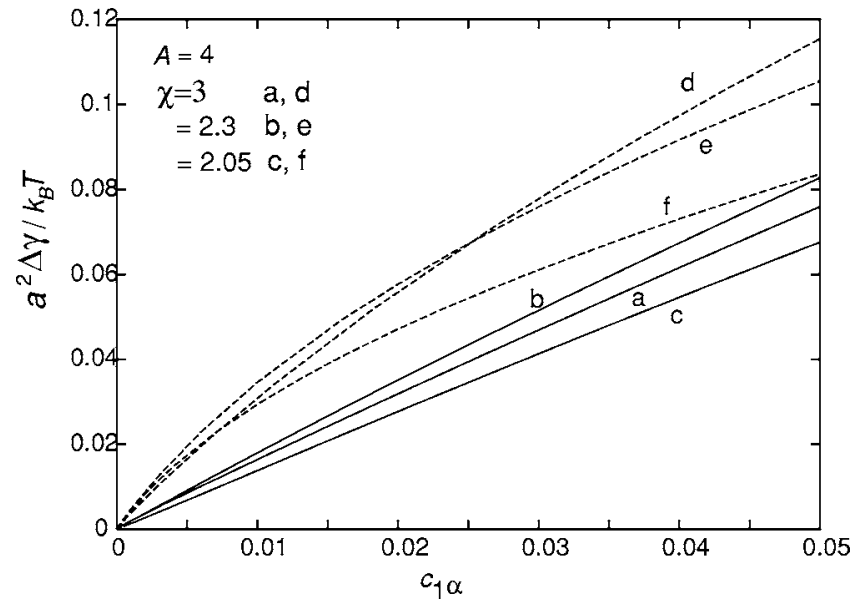

FIG. 6. Normalized excess surface tension $a^{2} \Delta \gamma / k_{B} T$ vs $c_{1 \alpha}$ for $\chi=3$ (a), 2.3 (b), and 2.05 (c) with $g_{1}=4$ and $g_{2}=2$ (solid lines), and for $\chi=3(\mathrm{~d}), 2.3(\mathrm{e})$, and 2.05 (f) with $g_{1}=10$ and $g_{2}=5$ (broken lines).

which $a^{2} \gamma_{0} / k_{B} T=0.498,0.103$, and 0.0773 , respectively. Here $\gamma_{0}$ are very different for these values of $\chi$. We set $g_{1}$ $=4$ and $g_{2}=2$ for the curves (a), (b), and (c) (solid lines), while $g_{1}=10$ and $g_{2}=5$ for the curves (d), (e), and (f) (broken lines). We generally find that $\Delta \gamma$ increases with increasing $g_{i}$. The linear dependence $\Delta \gamma \propto c_{1 \alpha}$ roughly holds at relatively large $c_{1 \alpha}$, but the curves depend on the various parameters in a rather complicated manner at smaller $c_{1 \alpha}$. The characteristic length $\lambda_{\mathrm{s}}$ defined by Eq. (1.3) is obtained from $\lambda_{\mathrm{s}} / a$ $=\left(a^{2} \Delta \gamma / k_{B} T\right) / c_{1 \alpha}$. Then we can see that $\lambda_{\mathrm{s}} / a \sim 2$ for the curves in Fig. 6.

\section{E. Approximations in dilute electrolytes}

When $n_{1}$ and $n_{2}$ are very small, we set

$$
\phi(z)=\phi_{0}(z)+\delta \phi(z)
$$

where the deviation $\delta \phi(z)$ is assumed to be small. From Eq. (4.31) and the relation $f^{\prime}\left(\phi_{0 K}\right)=0$ the deviations

$$
f\left(\phi_{K}\right)-f_{0}=\frac{1}{2} k_{B} \operatorname{Tr}_{K}\left(\phi_{K}-\phi_{0 K}\right)^{2}+\cdots
$$

at $z= \pm \infty$ are of second order in $\delta \phi$, where

$$
r_{K}=f^{\prime \prime}\left(\phi_{0 K}\right) / k_{B} T
$$

For our $f$ in Eq. (2.14), we have $r_{\alpha}=r_{\beta}$, as can be seen from Eq. (A2). Now Eq. (4.25) gives

$$
h \cong-k_{B} T \Delta n / \Delta \phi,
$$

where $\Delta n$ is the ion-density difference,

$$
\Delta n=n_{\alpha}-n_{\beta}=n_{\alpha}\left[1-\exp \left(-\epsilon_{r} / k_{B} T\right)\right]
$$

From Eq. (4.26), the composition deviations far from the interface are written as 


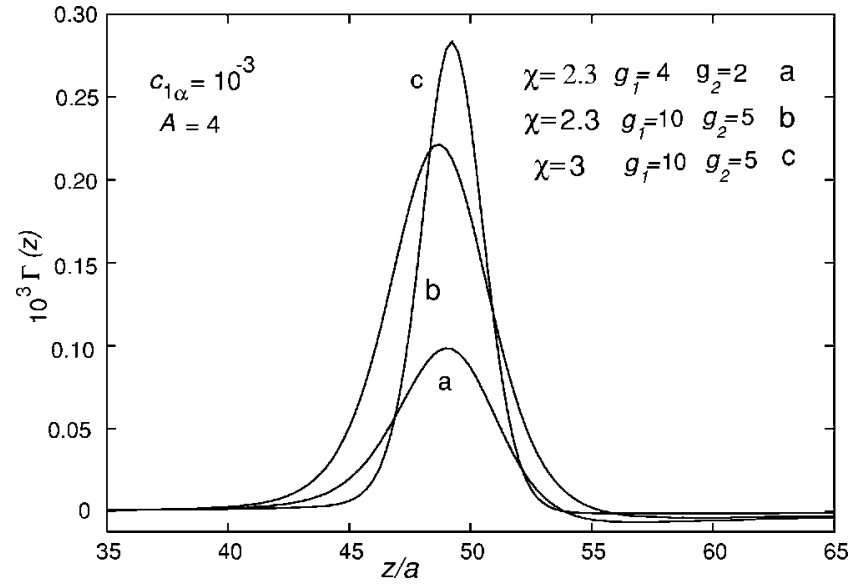

FIG. 7. Normalized integrand $\Gamma(z)$ of Eq. (4.41) for $\chi=2.3, g_{1}$ $=4$, and $g_{2}=2$ (a), for $\chi=2.3, g_{1}=10$, and $g_{2}=5$ (b), and for $\chi=3$, $g_{1}=10$, and $g_{2}=5$ (c). Its integral with respect to $z / a$ is equal to $a^{2} \Delta \gamma / k_{B} T$ in the approximate formula (4.40).

$$
\phi_{K}-\phi_{0 K} \cong \frac{1}{r_{K}}\left[g_{1} n_{1 K}+g_{2} n_{2 K}-\frac{\Delta n}{\Delta \phi}\right],
$$

where $K=\alpha, \beta$. This relation is consistent with the results in Fig. 5.

We next devise an approximate formula for $\Delta \gamma$ in the dilute case starting with Eq. (4.29). From Eq. (4.31), we have

$$
f(\phi)+\frac{C}{2}\left|\phi^{\prime}\right|^{2}=f\left(\phi_{0}\right)+\frac{C}{2}\left|\phi_{0}^{\prime}\right|^{2}+C \frac{d}{d z}\left(\phi_{0}^{\prime} \delta \phi\right)+\cdots,
$$

where the contributions of second order in $\delta \phi$ are not written. Since the third term on the right-hand side of Eq. (4.39) vanishes on integration, we obtain

$$
\frac{\Delta \gamma}{k_{B} T} \cong \int d z\left[n_{\alpha}-n(z)-\frac{\Delta n}{\Delta \phi}\left(\phi_{\alpha}-\phi(z)\right)\right]
$$

to linear order in $\delta \psi$. The integrand tends to zero for large $|z|$.

We introduce the normalized integrand of Eq. (4.40)

$$
\Gamma(z)=v_{0}\left(n_{\alpha}-n(z)\right)-v_{0}(\Delta n / \Delta \phi)\left(\phi_{\alpha}-\phi(z)\right) .
$$

In Fig. 7, we show $\Gamma(z)$ for three cases with $A=4$ and $c_{1 \alpha}$ $=10^{-3}$. It is positive mostly, but has a small negative tail in the phase $\beta$ (in the region $z / a \gtrsim 55$ here). Numerical values of $\lambda_{\mathrm{s}} / a$ are 1.84 from Eq. (4.29) and 1.96 from Eq. (4.40) for the curve (a) with $\chi=2.3, g_{1}=4$, and $g_{2}=2$. They are 4.86 and 4.62 for (b) with $\chi=2.3, g_{1}=10$, and $g_{2}=5$, and are 3.86 and 3.68 for (c) with $\chi=3, g_{1}=10$, and $g_{2}=5$, respectively. The differences between these two values are of order $5 \%$ at $c_{1 \alpha}=10^{-3}$; thus, the approximate formula (4.40) is not very precise at this ion density. Increases in $\lambda_{s}$ or $\Delta \gamma$ with increasing $g_{1}$ and $g_{2}$ are again observed. However, for $g_{1}=10$ and $g_{2}=5, \lambda_{s}$ is larger for (b) with $\chi=2.3$ than for (c) with $\chi=3$, since $\Gamma(z)$ is more broadened for (b) than for (c) in Fig. 7. The same tendency can also be found between the two curves (a) and (b) and between the two curves (d) and (e) at small $c_{1 \alpha}$ in Fig. 6. Additional comments are as follows. (i)

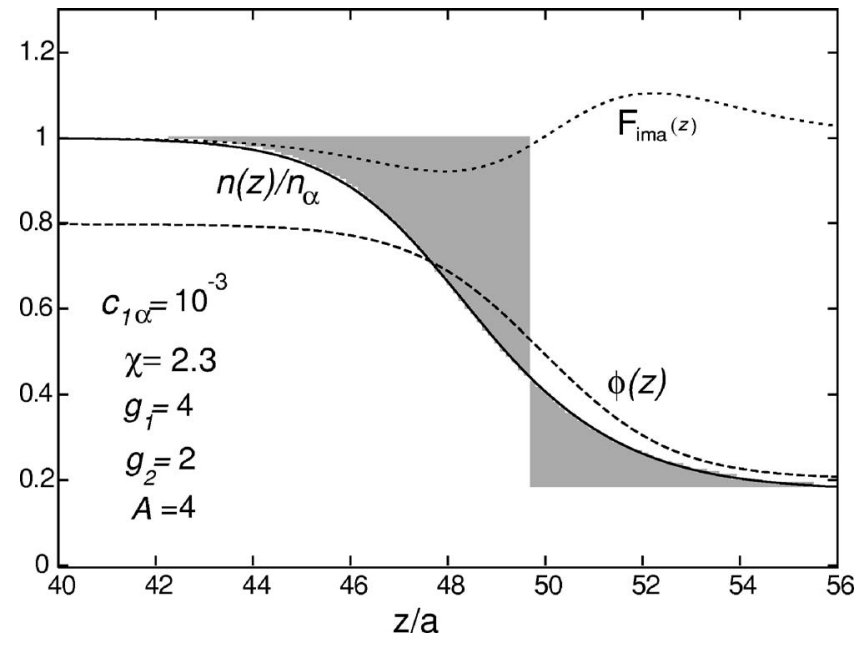

FIG. 8. Normalized ion density $n(z) / n_{\alpha}$, composition $\phi(z)$, and image factor $F_{\text {ima }}(z)$ for $\chi=2.3, g_{1}=4, g_{2}=2$, and $c_{1 \alpha}=10^{-3}$. The gray regions correspond to the two terms in Eq. (4.45).

The contributions from the integral $-\int \rho \Phi / 2=-\int d z \varepsilon \boldsymbol{E}^{2} / 8 \pi$ in Eq. (4.29) are at most a few percent of the total contribution in all the calculations. (ii) In the right-hand sides of Eqs. (4.29) and (4.40), we have used the same $\phi$ and $n_{1 K}$ for finite ion densities.

\section{F. Repulsion of ions from an interface}

It is convenient to define the interface position $z_{\text {int }}$ by

$$
\phi_{\alpha} z_{\text {int }}+\phi_{\beta}\left(L-z_{\text {int }}\right)=\int_{0}^{L} d z \phi(z)
$$

which is the Gibbs construction of the interface position [43]. We use the boundary condition (4.13), which allows the neglecting of the wetting layer. When the distances $z_{\text {int }}$ and $L-z_{\text {int }}$ are much longer than the interface thickness and the screening length, Eq. (4.40) may be expressed as

$$
\frac{\Delta \gamma}{k_{B} T} \cong \int_{-\infty}^{z_{\mathrm{int}}} d z\left[n_{\alpha}-n(z)\right]+\int_{z_{\mathrm{int}}}^{\infty} d z\left[n_{\beta}-n(z)\right],
$$

where the lower and upper bounds are pushed to infinity. Here, the first term is positive and the second term is negative, with the sum being positive, in our calculations. The second term becomes negligible for small $n(z)$ in the $\beta$ region $z>z_{\text {int }}$. This occurs in strong ion segregation with considerably large $g_{1}$ and $g_{2}$. The characteristic length $\lambda_{s}$ in Eq. (1.3) is then the thickness of the effective ion-depletion layer in the $\alpha$ region $z<z_{\text {int }}$. In the previous literature, only the first term in Eq. (4.43) has been considered in the thin interface limit $\xi \rightarrow 0$.

We illustrate how the two terms in Eq. (4.43) can be interpreted graphically. In Figs. 8-10, we display the normalized ion density $n(z) / n_{\alpha}$, the composition $\phi(z)$, and the image factor defined by

$$
F_{\text {ima }}(z)=\exp \left[-\mu_{\text {im }}(z) / k_{B} T\right],
$$

which appears in $n_{1}$ and $n_{2}$ in Eq. (2.30) or Eq. (C2) in the monovalent case. In these figures, the areas of the left and 


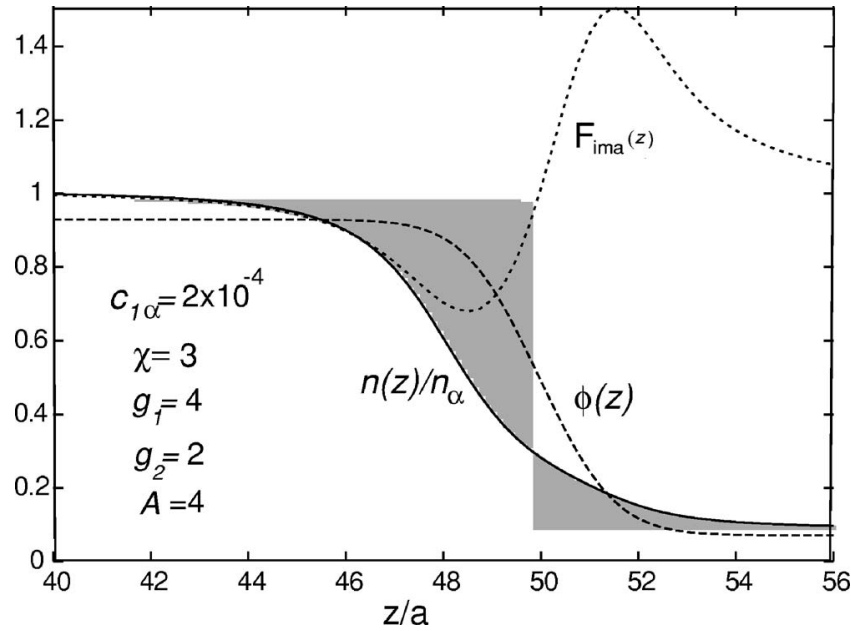

FIG. 9. Normalized ion density $n(z) / n_{\alpha}$, composition $\phi(z)$, and image factor $F_{\text {ima }}(z)$ for $\chi=3, g_{1}=4, g_{2}=2$, and $c_{1 \alpha}=2 \times 10^{-4}$.

right gray regions divided by $a^{2}$ are equal to the first term and the negative of the second term in Eq. (4.43), respectively. The ion density $n(z)$ is shifted to the left of the interface at $z=z_{\text {int }} \cong 50 a$. This means that the ions are repelled from the interface in the phase $\alpha$.

We furthermore mention detailed characteristic features. (i) In Fig. 8, we set $\chi=2.3, c_{1 \alpha}=10^{-3}, g_{1}=4, g_{2}=2$, and $A$ $=4$, where the ion density is relatively large and the ion reduction factor $\left(\sim e^{-1.8}\right)$ is not very small. The first term in Eq. (4.43) is then $158 \%$ of the total $\Delta \gamma / k_{B} T=2.03 a^{-2} c_{1 \alpha}$, while the second term is $-58 \%$. In this case, $F_{\text {ima }}(z) \cong 1$ at any $z$ so that the image interaction is not important. (ii) In Fig. 9, we set $\chi=3, c_{1 \alpha}=2 \times 10^{-4}, g_{1}=4, g_{2}=2$, and $A=4$, where $\chi$ is increased and $c_{1 \alpha}$ is decreased. The first term of Eq. (4.43) is $138 \%$ of the total $\Delta \gamma / k_{B} T=2.55 a^{-2} c_{1 \alpha}$ and the image interaction causes considerable reduction of $n(z)$. (iii) In Fig. 10, we set $\chi=3, c_{1 \alpha}=2.1 \times 10^{-5}, g_{1}=10, g_{2}=5$, and $A=10$, where $g_{i}$ and $A$ are increased, the ion density is very

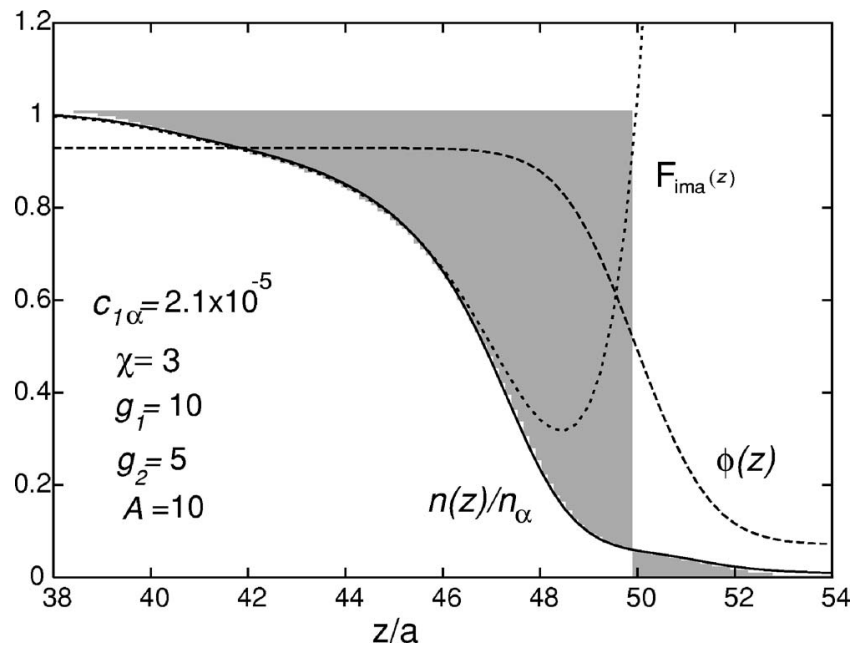

FIG. 10. Normalized ion density $n(z) / n_{\alpha}$, composition $\phi(z)$, and image factor $F_{\text {ima }}(z)$ for $\chi=3, g_{1}=10, g_{2}=5$, and $c_{1 \alpha}=2.1 \times 10^{-5}$. For these parameters, the role of the image factor on $n(z)$ is more dominant than that of the solvation interaction. small, and $1 / \kappa_{\alpha}=13.3$ is rather long. The first term of Eq. (4.43) is $103.5 \%$ of the total $a^{2} \Delta \gamma / k_{B} T=7.32 c_{1 \alpha}$ and the role of the image factor is much intensified.

We discuss the relation between our theory and the Onsager-Samarus theory [25]. For $z_{\text {int }}-z \gtrsim \xi$, the image factor behaves as

$$
F_{\text {ima }}(z) \cong \exp \left[-D_{\mathrm{I}} e^{\left.-2 \kappa_{\alpha} \mid z-z_{\text {int }} /\left(z_{\text {int }}-z\right)\right],}\right.
$$

where $D_{\mathrm{I}}=a A \varepsilon_{1} \Delta \phi / \pi \varepsilon_{c}$ in our approximation. The image interaction can be important in the $\alpha$ region for $D_{\mathrm{I}} \gtrsim \xi$ or

$$
A \varepsilon_{1} \Delta \phi / \varepsilon_{c} \gtrsim \xi / a,
$$

under which $F_{\text {ima }}(z)$ is considerably smaller than unity at $z_{\text {int }}-z \sim \xi$. If $\varepsilon_{1} / \varepsilon_{c}, \Delta \phi$, and $\xi / a$ are of order unity away from the critical point, this condition becomes $A \sim \ell_{\mathrm{B} c} / a$ $\gtrsim 1$. Moreover, if $D_{\mathrm{I}} \kappa_{\alpha} \ll 1$ in the dilute limit, the integral of $n_{\alpha}-n(z)$ in the region $D_{\mathrm{I}}<z_{\text {int }}-z<1 / \kappa_{\alpha}$ becomes $n_{\alpha} D_{\mathrm{I}} \ln \left(1 / D_{\mathrm{I}} \kappa_{\alpha}\right)$ and that in the region $\xi<z_{\text {int }}-z<D_{\mathrm{I}}$ is of order $n_{\alpha} D_{\mathrm{I}}$. Thus,

$$
\Delta \gamma / k_{B} T \cong n_{\alpha} D_{\mathrm{I}}\left[\ln \left(1 / D_{\mathrm{I}} \kappa_{\alpha}\right)+E_{\mathrm{I}}\right],
$$

where $E_{\mathrm{I}}$ is a dimensionless constant of order unity. If $D_{\mathrm{I}}$ is set equal to the classical value $e^{2} / 4 \varepsilon_{\alpha} k_{B} T$ in the case $\varepsilon_{\alpha}$ $\gg \varepsilon_{\beta}$, the above result reduces to that by Onsager and Samaras (see Appendix C).

\section{G. Approaching criticality in two-phase states}

We are interested in the coexistence curve in the presence of ions in the $\phi-T(\phi-\chi)$ plane [44-46]. We assume the stability of the system against formation of a mesoscopic phase or $\gamma_{p}<1$ (see Appendix A) [32,39]. We seek the solution of Eqs. (4.25) and (4.26) supplemented with Eq. (4.10) to calculate $\psi_{\alpha}=\phi_{\alpha}-1 / 2$ and $\psi_{\beta}=\phi_{\beta}-1 / 2$. As a parameter representing the amount of the doped ions, there can be many choices. It is convenient to introduce an ion density [50],

$$
\bar{n}=\left(n_{\alpha} n_{\beta}\right)^{1 / 2} .
$$

In the monovalent case, we have $v_{0} \bar{n}=2\left(c_{1 \alpha} c_{1 \beta}\right)^{1 / 2}$ in the dimensionless form so that $n_{1 \alpha}=\exp \left(\epsilon_{\mathrm{r}} / 2 k_{B} T\right) \bar{n} / 2$ and $n_{1 \beta}$ $=\exp \left(-\epsilon_{\mathrm{r}} / 2 k_{B} T\right) \bar{n} / 2$. We treat $\bar{n}$ and $2-\chi$ as small control parameters characterizing two-phase states with a macroscopic planar interface. If the system is close to the critical point, we may use the Landau expansion $f / k_{B} T=\tau \psi^{2} / 2$ $+u \psi^{4} / 4+$ const., where our free energy density Eq. (2.14) gives

$$
\tau=(4-2 \chi) / v_{0}, \quad u=16 / 3 v_{0} .
$$

We also expand the ion reduction factor in Eq. (4.10) in powers of $\epsilon_{\mathrm{r}} / k_{B} T \propto \Delta \phi=\psi_{\alpha}-\psi_{\beta}$. Some calculations show that the critical condition $\Delta \phi \rightarrow 0$ is attained at $\tau=\tau_{c}^{\text {ion }}$ with

$$
\tau_{c}^{\text {ion }}=\left[\left(g_{1} Z_{2}+g_{2} Z_{1}\right) /\left(Z_{1}+Z_{2}\right)\right]^{2} \bar{n} .
$$

Here, $\chi_{c}^{\text {ion }}=2-\tau_{c}^{\text {ion }} v_{0} / 2$ and $\bar{n}=\langle n\rangle(=$ space average $)$ at the critical point, so the above relation is consistent with Eq. (A5). If $\tau$ is slightly smaller than $\tau_{c}^{\text {ion }}, \tau-\tau_{c}^{\text {ion }}$ represents the reduced temperature at fixed $\bar{n}$ and that $\psi_{\alpha}$ and $\psi_{\beta}$ behave as 


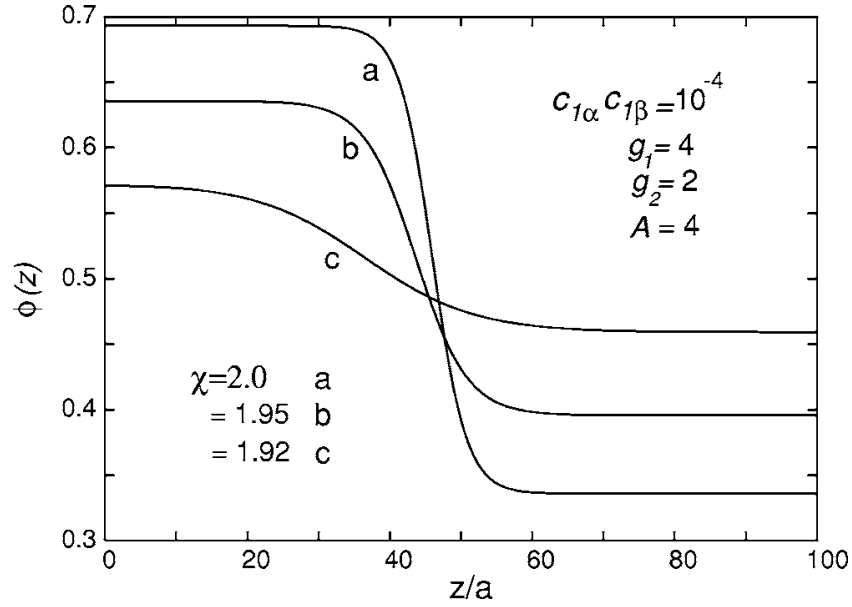

FIG. 11. Composition profiles with varying $\chi$ for $g_{1}=4, g_{2}=2$, $A=4$, and $v_{0} \bar{n}=0.02$, for which $\chi_{c}^{\text {ion }}=1.91$.

$$
\begin{gathered}
\psi_{\alpha}+\psi_{\beta} \cong\left[\left(g_{1} Z_{2}+g_{2} Z_{1}\right) /\left(Z_{1}+Z_{2}\right)\right]^{3} \frac{\bar{n}}{3 u}, \\
\Delta \phi=\psi_{\alpha}-\psi_{\beta} \cong 2\left[\left(\tau_{c}^{\text {ion }}-\tau\right) / u\right]^{1 / 2} .
\end{gathered}
$$

The first line is the shift of the critical composition multiplied by 2 . The second line is the usual mean-field expression.

We next approach the critical point numerically with decreasing $\chi$ in two-phase states. As an example, let us set $g_{1}$ $=4, g_{2}=2, A=4$, and $v_{0} \bar{n}=0.02$. Then the parameter $\gamma_{p}$ in Eq. (A6) becomes 0.29 and the critical value of $\chi$ becomes $\chi_{c}^{\text {ion }}$ $=2-4.5\left\langle c_{1}+c_{2}\right\rangle=1.91$ from Eq. (4.50). In Fig. 11, we show the profiles of $\psi(z)$ for $\chi=2,1.95$, and 1.92 or for $\chi_{c}^{\text {ion }}-\chi$ $=0.09,0.04$, and 0.01 as the curves (a), (b), and (c). Evidently, the interface thickness increases as $\chi \rightarrow \chi_{c}^{\text {ion }}$. For these cases, Eqs. (4.51) and (4.52) yield $\psi_{\alpha}+\psi_{\beta}=0.0375$ and $\Delta \phi$ $=1.22\left(\chi^{\text {ion }}-\chi\right)^{1 / 2}$ with the aid of Eq. (4.49). For (a), (b), and (c), numerical values are $\psi_{\alpha}+\psi_{\beta}=0.0291,0.0314$, and 0.0303 and $\Delta \phi /\left(\chi^{\text {ion }}-\chi\right)^{1 / 2}=1.19,1.20$, and 1.12 , respectively. Therefore, Eqs. (4.51) and (4.52) nicely hold, although $n_{\alpha}$ and $n_{\beta}$ are considerably different for (a) and (b). On the other hand, the normalized potential difference $\Delta U$ $=e\left(\Phi_{\alpha}-\Phi_{\beta}\right) / k_{B} T$ is $0.357,0.240$, and 0.112 , respectively, in agreement with Eq. (4.8). For $\chi<1.91$ there can only be a solution representing a uniform one-phase state. In Fig. 12, we show $c_{1}(z)$ and $c_{1}(z)-c_{2}(z)$. When the interface thickness $\xi$ much exceeds the screening length, the ion distributions have transition regions with a thickness of order $\xi$, as it should be the case. The electric double layer at the interface diminishes on approaching the criticality. In addition, the surface tension exhibits the usual mean-field behavior $\gamma$ $\propto\left(\chi-\chi^{\text {ion }}\right)^{3 / 2}$ near the ion-induced criticality in the meanfield theory.

\section{H. Slow decay of electric potential at small $\kappa_{\beta}$}

When the ion density is very small in the phase $\beta$, the potential $\Phi(z)$ slowly changes over the distance of $1 / \kappa_{\beta}$ in the region $z>z_{\text {int }}$. Figure 13 displays $U(z)=e \Phi(z) / k_{B} T$ at

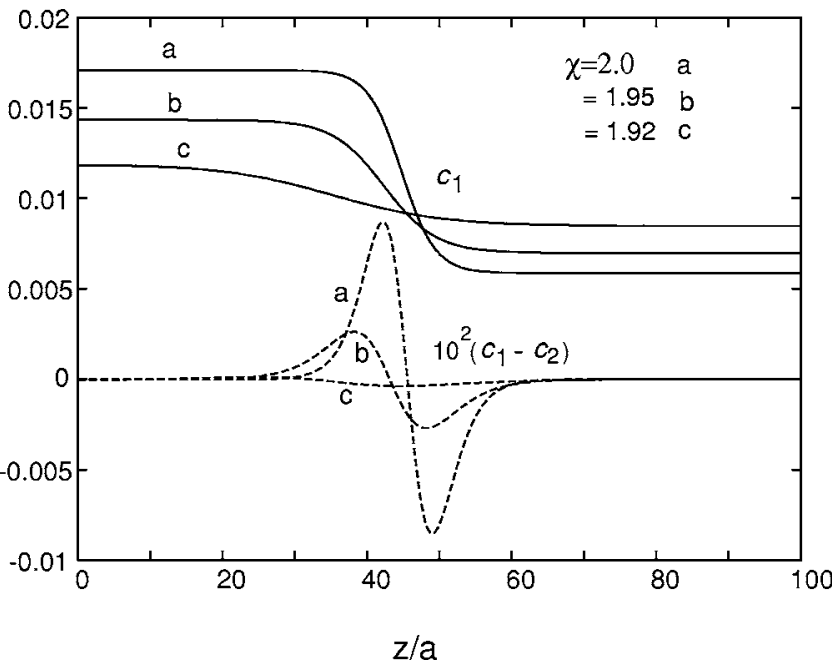

FIG. 12. Normalized ion density $c_{1}(z)$ and normalized charge density $c_{1}(z)-c_{2}(z)$ (multiplied by 100) with varying $\chi$ with the same parameter values as in Fig. 11.

$c_{1 \alpha}=5 \times 10^{-4}$ with $\chi=2.3, g_{1}=10, g_{2}=5$, and $A=4$. We use the boundary conditions in Eq. (4.13) with $L=200 a$. Since $\phi_{\alpha}=0.800$ and $\phi_{\beta}=0.203$, the ion reduction factor in Eq. (4.10) is 0.012 , and $c_{1 \beta}=5.9 \times 10^{-6}$, resulting in $1 / \kappa_{\alpha}$ $=5.07 \mathrm{a}$ and $1 / \kappa_{\beta}=40.0 \mathrm{a}$.

In the $\beta$ region not close to the interface $\left(z-z_{\text {int }} \gg \xi\right)$, the composition deviation $\psi(z)-\psi_{\beta}$ may be neglected and the normalized potential obeys

$$
U^{\prime \prime}(z)=\kappa_{\beta}^{2} F_{\text {ima }}(z) \sinh \left(U(z)-U_{\beta}\right)
$$

where $U^{\prime \prime}=d^{2} U / d z^{2}$ and $U_{\beta}=e \Phi_{\beta} / k_{B} T$. The image factor defined in Eq. (4.44) behaves as

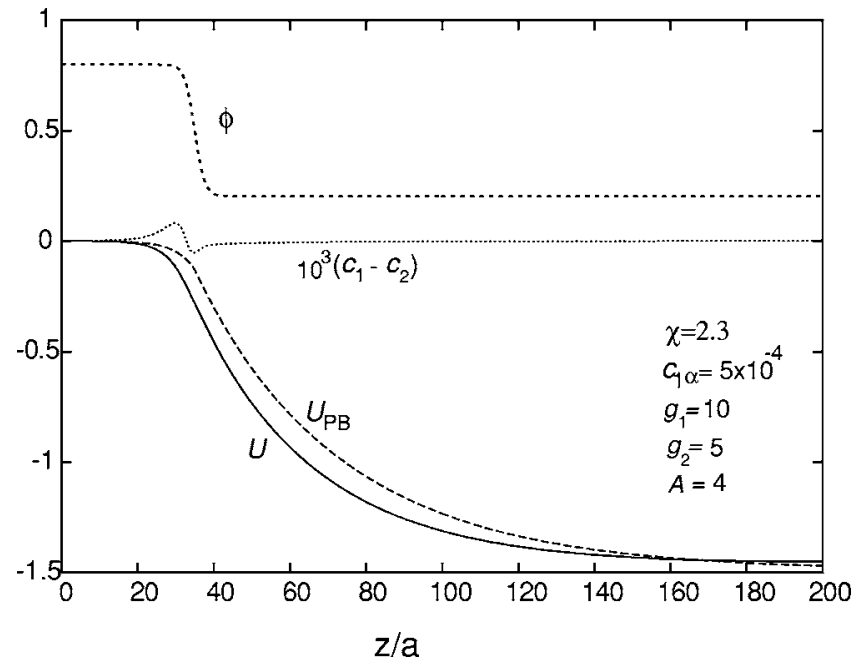

FIG. 13. Normalized electric potential $U(z)=e \Phi(z) / k_{B} T$ (solid line) at small ion density $c_{1 \alpha}=5 \times 10^{-4}$ with $\chi=2.3, g_{1}=10, g_{2}=5$, and $A=4$. Here $c_{1 \beta}=5.9 \times 10^{-6}$ and $\kappa_{\beta}=2.5 \times 10^{-2}$. It is compared with the Poisson-Boltzmann solution, Eqs. (4.55) and (4.56), without the image interaction. Also shown are composition profile $\phi(z)$ and the scaled charge density $10^{3}\left[c_{1}(z)-c_{2}(z)\right]$. 


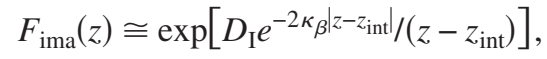

where $D_{\mathrm{I}}=a A \varepsilon_{1} \Delta \phi / \pi \varepsilon_{c}$, as in Eq. (4.45), and $D_{I}=0.61 a$ in Fig. 13. For example, this factor is equal to 1.038 at $z-z_{\text {int }}$ $=10 a$ in Fig. 13 .

If the image factor is set equal to unity, Eq. (4.53) becomes the well-known Poisson-Boltzmann equation which neglects the solvation and image interactions. Its solution, written as $U_{\mathrm{PB}}^{>}$, is given by

$$
U_{\mathrm{PB}}^{>}(z)=U_{\beta}+2 \ln \left[\frac{1+d_{\beta} e^{-\kappa_{\beta}\left|z-z_{\mathrm{int}}\right|}}{1-d_{\beta} e^{-\kappa_{\beta}\left|z-z_{\mathrm{int}}\right|}}\right],
$$

in the region $z>z_{\text {int }}$. If $\beta$ is changed to $\alpha$ in Eq. (4.55), the Poisson-Boltzmann solution is also obtained in the $\alpha$ region, which is written as $U_{\mathrm{PB}}^{<}$. We require the continuity of the electric potential and the electric induction, $U_{\mathrm{PB}}^{>}=U_{\mathrm{PB}}^{<}$and $\varepsilon_{\beta} d U_{\mathrm{PB}}^{>} / d z=\varepsilon_{\alpha} d U_{\mathrm{PB}}^{<} / d z$, at $z=z_{\text {int }}$. We then connect the two one-sided solutions,

$$
\begin{aligned}
& U_{\mathrm{PB}}(z)=U_{\mathrm{PB}}^{>}(z) \quad\left(z>z_{\text {int }}\right) \\
& =U_{\mathrm{PB}}^{<}(z) \quad\left(z<z_{\text {int }}\right),
\end{aligned}
$$

which decreases from $U_{\alpha}$ at $z=-\infty$ to $U_{\beta}$ at $z=\infty$ by $\Delta U$ $=e\left(\Phi_{\alpha}-\Phi_{\beta}\right) / k_{B} T$. In Fig. 13, we plot $U_{\mathrm{PB}}$ thus obtained with $d_{\beta}=0.328$. If $\varepsilon_{\beta} \kappa_{\beta} / \varepsilon_{\alpha} \kappa_{\alpha} \ll 1, d_{\beta}$ and $U_{\alpha}-U_{\mathrm{PB}}\left(z_{\text {int }}\right)$ are given by $\sinh (\Delta U / 4)$ (close to the above value) and the first term on the right-hand side of Eq. (4.20) multiplied by $k_{B} T / e$, respectively.

Comparing $U$ and $U_{\mathrm{PB}}$, we recognize the following. (i) In the $\beta$ region, $U$ relaxes to $U_{\beta}$ slightly faster than $U_{\mathrm{PB}}$ due to the image factor in Eq. (4.54). (ii) The curve of $U$ is shifted into the $\alpha$ region, as compared to $U_{\mathrm{PB}}$, and the decrease of $U$ in the $\alpha$ region $U_{\alpha}-U\left(z_{\text {int }}\right)$ is considerably larger than that of $U_{\mathrm{PB}}$ in Eq. (4.56). These are due to the charge density created by the asymmetric solvation interaction near the interface, [see Eqs. (4.19) and (4.20)]. (iii) Nevertheless, the small negative tail of the charge density $\rho(z)\left(\cong-\varepsilon_{\beta} \Phi^{\prime \prime} / 4 \pi\right)$ in the $\beta$ region (at least for $z-z_{\text {int }} \geq 1 / \kappa_{\beta}$ ) is correctly described by the Poisson-Boltzmann theory.

In Fig. 14, we plot the potential reduction ratio $R_{p}$ $\equiv\left[\Phi_{\alpha}-\Phi\left(z_{\text {int }}\right)\right] /\left(\Phi_{\alpha}-\Phi_{\beta}\right)=\left[U_{\alpha}-U\left(z_{\text {int }}\right)\right] / \Delta U$ in the $\alpha$ region as a function of $c_{1 \alpha}$ with $A=4$ for three cases. It is around 0.4 for weak ion segregation at $\chi=2.3, g_{1}=4$, and $g_{2}=2$ on the curve (a). However, it decreases with decreasing $c_{1 \alpha}$ for strong ion segregation with $g_{1}=10$ and $g_{2}=5$ on the curves (b) and (c).

\section{Hydrophilic and hydrophobic ions}

If hydrophilic and hydrophobic ions are in aqueous solutions, we have $\Delta \mu_{\alpha \beta}^{1}>0$ and $\Delta \mu_{\alpha \beta}^{2}<0[14,17]$ or $g_{1}>0$ and $g_{2}<0$ under Eq. (2.19). With decreasing $g_{2}$, the potential difference (4.8) becomes larger and $\epsilon_{r}$ in Eq. (4.10) can even change its sign for this case. In Fig. 15, we set $g_{1}=4$ and $g_{2}=-4$ with $c_{1 \alpha}=10^{-3}$, where $\epsilon_{r}=0$ and the bulk ion densities far from the interface coincide. We can see that $c_{1}$ exhibits a maximum in the $\alpha$ region and a minimum in the $\beta$ region, while $c_{2}$ has extremums in the reverse regions. Thus the relative charge density $\left(c_{1}-c_{2}\right) / c_{1 \alpha}$ is more intensified than

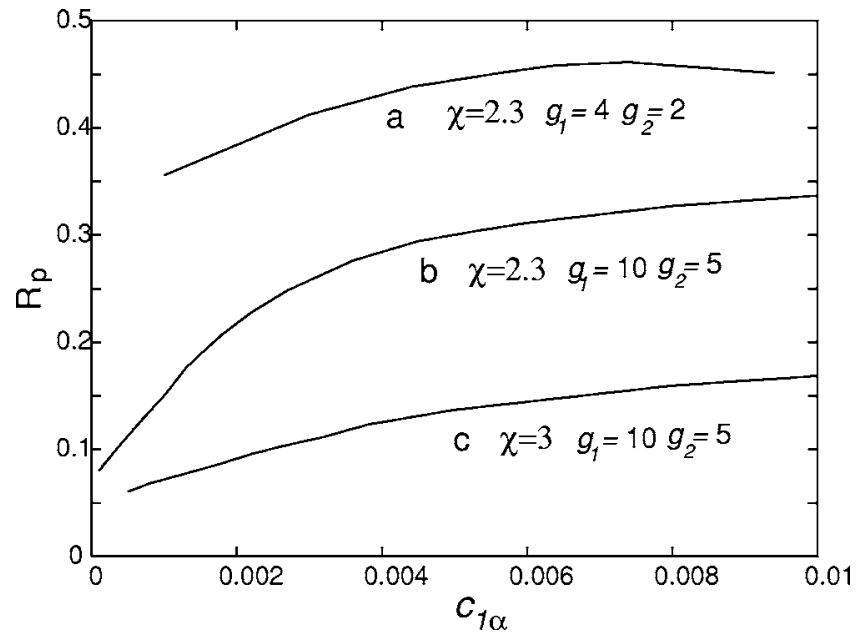

FIG. 14. Potential reduction ratio in the $\alpha$ region $R_{p}=\left[\Phi_{\alpha}\right.$ $\left.-\Phi\left(z_{\text {int }}\right)\right] /\left(\Phi_{\alpha}-\Phi_{\beta}\right)$ versus $c_{1 \alpha}$, where $\chi=2.3, g_{1}=4$, and $g_{2}=2$ (a), $\chi=2.3, g_{1}=10$, and $g_{2}=5$ (b), and $\chi=3, g_{1}=10$, and $g_{2}=5$ (c).

in the case of $g_{1}>g_{2}>0$. Interestingly, the integral of the deviation $c_{1}+c_{2}-2 c_{1 \alpha}$ is positive due to the image interaction in the $\beta$ region in this case. That is, the ions are absorbed onto the interface on the average and $\lambda_{s}$ in Eq. (1.3) becomes $-3.54 a$.

\section{SUMMARY AND REMARKS}

We have introduced the solvation effects at low ion densities into electrolyte theory in the simplest manner, neglecting ion association [2]. We have then examined the ion distributions, the electric potential, and the composition profile around an interface in polar binary mixtures in the GinzburgLandau scheme. We have derived a number of general relations. They are worth experimental investigation particularly near the critical point. Numerical calculations have been per-

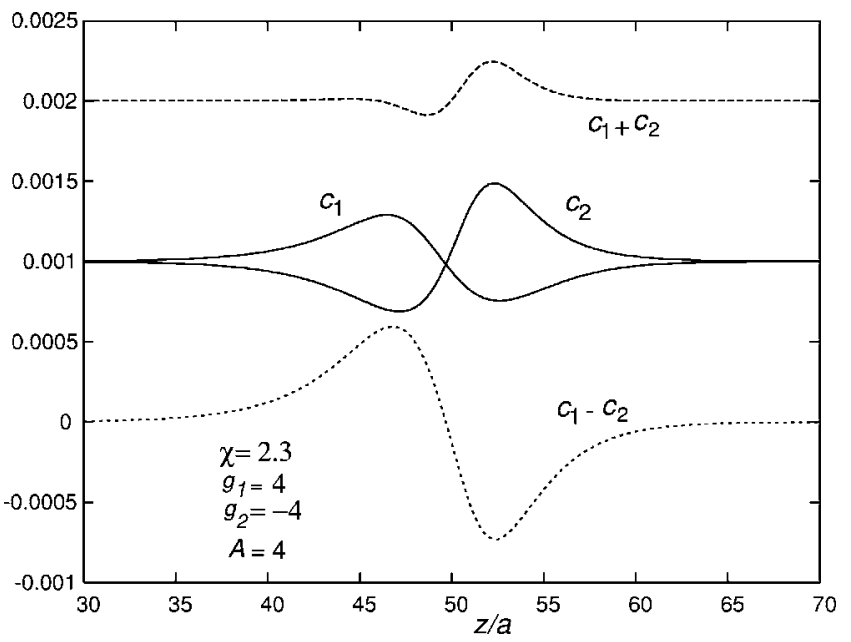

FIG. 15. Normalized ion densities $c_{1}$ and $c_{2}$ around an interface, where $g_{1}=4, g_{2}=-4, \chi=2.3$, and $A=4$. The first species is hydrophilic and the second one is hydrophobic. Also shown are $c_{1}-c_{2}$ $=a^{3} \rho / e$ and $c_{1}+c_{2}=a^{3} n$. A marked electric double layer is formed here. 
formed in one dimension and in the monovalent case only.

The ingredients of our theory are as follows.

(i) The dielectric constant in Eq. (2.4) is dependent on the composition and is inhomogeneous. Though we have treated a moderate case of $\varepsilon_{1} / \varepsilon_{0}=4 / 3$, the ratio $\varepsilon_{1} / \varepsilon_{0}$ can be of order 80 in aqueous solutions. Such very strong composition dependence should lead to various effects not well recognized so far. We mention a paper pointing out its relevance in first-order swelling transition of charged gels [51].

(ii) The solvation effects are taken into account in Eq. (2.13) with the composition-dependent solvation chemical potentials in Eq. (2.19). The resultant interaction in the free energy $F$ is bilinear and characterized by dimensionless parameters $g_{i}$ dependent on the ion species $i$. A shift of the coexistence curve of the composition follows from this coupling [32]. Intriguing is the asymmetric case $g_{1} \neq g_{2}$, where a potential difference arises at an interface. In one-phase states, this asymmetry can lead to a peak in the structure factor of the composition fluctuations at an intermediate wave number, as summarized in Appendix A. There can even be a mesoscopic phase [39], which can possibly occur in the case of strongly asymmetric salt with large $g_{1}-g_{2}$ (say, salt composed of small cations and relatively large anions with distinctly different solvation powers) [32]. Notice that $g_{i}$ can be negative for hydrophobic ions. Also we stress that the critical fluctuations give rise to attractive interactions among the ions as shown in Eqs. (A7) and (A8), whose physical consequences should be examined in future.

(iii) The image interaction is shown to arise from general inhomogeneous dielectric constant and is expressed in the integral form in Eq. (2.21) or Eq. (3.7), though our expression can be used only for weak or moderate inhomogeneity. As discussed in Sec. III, it can dominate over the solvation interaction for $A \gg 1$, at very small ion densities, and far below the critical point.

The charge effects become very complex with these new ingredients and our present results are still fragmentary. We mention related problems.

(i) There can be an electric double layer and a potential difference at an interface in general charged systems, including low-molecular-weight fluids (as in this paper), complex fluids, gels, and even liquid metals. In particular, solvation effects in microemulsion systems and charged colloidal systems are of great interest.

(ii) Wetting transitions should be greatly influenced by ions $[52,53]$. If the wetting layer is more polar than the outer fluid, ions can even be confined in the layer for $\Delta \mu_{\alpha \beta}^{i}$ $\gg k_{B} T$ [see Eq. (1.2)].

(iii) We mention dynamical problems. Time evolution of the ion distributions is a slow process due to slow ion diffusion [19]. Phase-separation processes should also be influenced by ions which are more strongly segregated than the solvent composition for large $g_{i}$. We already examined the effect of a very small amount of ions on nucleation in a metastable fluid in a less polar phase. There, the nucleation barrier is decreased by $\Delta \mu_{\alpha \beta}^{i}$ in Eq. (1.2) for shallow quenching [22]. Ion-induced nucleation plays a decisive role in atmospheric phenomena.

(iv) Recently, we examined solvation effects of charged particles in liquid crystals $[33,54]$. In nematic states, the di- electric tensor is anisotropically dependent on the director orientation and, as a result, the electric field of ions distorts the orientation over long distances and sometimes create nanometer scale defects.

\section{ACKNOWLEDGMENTS}

We would like to thank K. Orzechowski, M. Doi, K. Kitamura, K. Yoshikawa, H. Seto, and J. F. Joanny for valuable discussions. This work was supported by Grants in Aid for Scientific Research and for the 21st Century COE "Center for Diversity and Universality in Physics" from the Ministry of Education, Culture, Sports, Science, and Technology of Japan.

\section{APPENDIX A: FLUCTUATIONS IN ONE-PHASE STATES}

We consider small plane-wave fluctuations in a nearcritical one-phase state, where the inhomogeneity in the dielectric constant can be neglected $\left(\varepsilon=\varepsilon_{\mathrm{c}}\right)$ to leading order. Retaining the Fourier components with wave numbers much larger than the inverse system size $1 / L$, we first write the fluctuation contributions to $F$ in the bilinear order as [32]

$$
\begin{aligned}
\delta F= & \int_{\mathbf{q}}\left[\frac{1}{2}\left(f^{\prime \prime}+C q^{2}\right)\left|\phi_{\mathbf{q}}\right|^{2}+\frac{2 \pi}{\varepsilon_{\mathrm{c}} q^{2}}\left|\rho_{\mathbf{q}}\right|^{2}+k_{B} T\right. \\
& \left.\times \sum_{i=1,2}\left(\frac{\left|n_{i \mathbf{q}}\right|^{2}}{2 n_{i}}-\left(g_{i}-Z_{i}^{2} I_{q}\right) n_{i \mathbf{q}} \phi_{\mathbf{q}}^{*}\right)\right],
\end{aligned}
$$

where $\int_{\mathbf{q}} \cdots=(2 \pi)^{-3} \int d \mathbf{q} \cdots$ denotes the integration over the wave vector $\mathbf{q}$ and

$$
f^{\prime \prime}=\frac{\partial^{2} f}{\partial \phi^{2}}=\frac{k_{B} T}{v_{0}}\left[\frac{1}{\phi(1-\phi)}-2 \chi\right]
$$

in our model. The correlation length is given by $\xi$ $=\left(C / f^{\prime \prime}\right)^{1 / 2}$ for the case without ions. The $\phi_{\mathbf{q}}, n_{i \mathbf{q}}$, and $\rho_{\mathbf{q}}$ are the Fourier transformations of $\phi(\boldsymbol{r}), n_{i}(\boldsymbol{r})$, and $\rho(\boldsymbol{r})$, respectively. The $n_{i}$ in the last term of Eq. (A1) are the average ion densities satisfying $Z_{1} n_{1}=Z_{2} n_{2}$ from the charge neutrality. The terms proportional to $I_{q}$ arise from the image interaction, where Eq. (2.24) yields

$$
I_{q}=\left(4 \pi B_{0} / k_{B} T\right) q \tan ^{-1}(q / 2 \kappa),
$$

so that $I_{q} \propto q^{2}$ for $q \ll \kappa$. The terms from the image interaction are negligible at any $q$ in one-phase states, so they will be omitted hereafter.

Elimination of the ion-density fluctuations yields the composition structure factor in the mean-field theory,

$$
\frac{k_{B} T}{S(q)}=f^{\prime \prime}-\Delta r_{\text {ion }}+C q^{2}\left[1-\frac{\gamma_{\mathrm{p}}^{2} \kappa^{2}}{\kappa^{2}+q^{2}}\right],
$$

where $\kappa$ is the Debye-Hückel wave number and $\Delta r_{\text {ion }}$ is the ion-induced shift,

$$
\Delta r_{\text {ion }}=k_{B} T\left(Z_{2} g_{1}+Z_{1} g_{2}\right)^{2} n /\left(Z_{1}+Z_{2}\right)^{2} .
$$

The shift becomes significant for large $g_{i}$ even for small $n$ $=n_{1}+n_{2}$. This is consistent with the experimental fact that 
even a small amount of salt greatly shifts the liquid-liquid coexistence curve [44-47]. The dimensionless parameter $\gamma_{\mathrm{p}}$ represents the asymmetry of the solvation in the two components,

$$
\begin{aligned}
\gamma_{\mathrm{p}} & =\left|g_{1}-g_{2}\right|\left(k_{B} T / 4 \pi C \ell_{B}\right)^{1 / 2} /\left(Z_{1}+Z_{2}\right) \\
& =\left|g_{1}-g_{2}\right| /\left[4\left(Z_{1}+Z_{2}\right) \sqrt{\chi^{A}}\right],
\end{aligned}
$$

where we have used Eqs. (2.18) and (3.3) in the second line. For $\gamma_{\mathrm{p}}>1, S(q)$ is maximum at an intermediate wave number and formation of a mesoscopic phase can be predicted below the transition point $[32,33,39]$. For $\gamma_{p}<1, S(q)$ is maximum at $q=0$ and the usual critical point still exists.

We may also eliminate the critical fluctuations in $F$ by setting $\left(f^{\prime \prime}+C q^{2}\right) \phi_{\mathbf{q}}=k_{B} T \sum_{i=1,2} g_{i} n_{i \mathbf{q}}$. The resultant free energy $F_{\text {ion }}$ for the ions only is expressed in the real space as

$$
\begin{aligned}
F_{\text {ion }}= & k_{B} T \int d \boldsymbol{r} \sum_{i} n_{i}\left[\ln \left(v_{0} n_{i}\right)-1\right]+\frac{1}{2} \int d \boldsymbol{r} \int d \boldsymbol{r}^{\prime} \\
& \times \sum_{i, j} V_{i j}\left(\left|\boldsymbol{r}-\boldsymbol{r}^{\prime}\right|\right) n_{i}(\boldsymbol{r}) n_{j}\left(\boldsymbol{r}^{\prime}\right),
\end{aligned}
$$

where the first entropic terms are not expanded with respect to the ion-density deviations and $V_{i j}(r)$ are the interaction potentials,

$$
V_{i j}(r)=Q_{i} Q_{j} \frac{1}{\varepsilon_{\mathrm{c}} r}-\frac{\left(k_{B} T\right)^{2}}{4 \pi C} g_{i} g_{j} \frac{e^{-r / \xi}}{r},
$$

with $Q_{1}=Z_{1} e$ and $Q_{2}=-Z_{2} e$. The second term in Eq. (A8) is the effective interaction mediated by the critical fluctuations. Among the ions of the same species $(i=j)$ it is attractive and dominates over the first Coulomb repulsive term in the intermediate range $a \lesssim r \lesssim \xi$ if

$$
g_{i}^{2}>4 \pi C Z_{i}^{2} e^{2} / \varepsilon_{\mathrm{c}}\left(k_{B} T\right)^{2} \sim 4 \pi Z_{i}^{2} \ell_{\mathrm{Bc}} / a .
$$

Under the above strong solvation condition, there should be a tendency of aggregation even among ions of the same species. This effect might explain a number of observations of microheterogeneities in near-critical binary mixtures containing salt [47].

\section{APPENDIX B: IMAGE POTENTIAL AND GENERALIZATION}

(i) First, let two fluid phases $\alpha$ and $\beta$ with dielectric constants $\varepsilon_{\alpha}$ and $\varepsilon_{\beta}$ be separated by a planar interface with the phase $\alpha$ in the region $z<0$. Here $\varepsilon_{\alpha}>\varepsilon_{\beta}$ and the thin interface limit is taken. We place an ion with charge $Q$ at a position $\boldsymbol{r}_{0}=\left(x_{0}, y_{0}, z_{0}\right)$ in the phase $\alpha\left(z_{0}<0\right)$. The resultant electric potential $\Psi$ should satisfy the surface condition,

$$
\varepsilon_{\alpha} \Psi^{\prime}(-0)=\varepsilon_{\beta} \Psi^{\prime}(+0),
$$

where $\Psi^{\prime}=d \Psi / d z$. Then, $\Psi$ in the phase $\alpha(z<0)$ is written as [49]

$$
\Psi\left(\boldsymbol{r}, \boldsymbol{r}_{0}\right)=\frac{Q}{\varepsilon_{\alpha}\left|\boldsymbol{r}-\boldsymbol{r}_{0}\right|}+\frac{\varepsilon_{\alpha}-\varepsilon_{\beta}}{\varepsilon_{\alpha}+\varepsilon_{\beta}} \frac{Q}{\varepsilon_{\alpha}\left|\boldsymbol{r}-\overline{\boldsymbol{r}}_{0}\right|},
$$

where $\overline{\boldsymbol{r}}_{0}=\left(x_{0}, y_{0},-z_{0}\right)$ is the image position in the phase $\beta$. The second term is produced by the polarization of the di- electric medium. As $\boldsymbol{r} \rightarrow \boldsymbol{r}_{0}$, it follows the repulsive potential of the ion dependent on $z_{0}(<0)$,

$$
\Phi_{\alpha}\left(z_{0}\right)=\frac{\varepsilon_{\alpha}-\varepsilon_{\beta}}{2\left(\varepsilon_{\alpha}+\varepsilon_{\beta}\right) \varepsilon_{\alpha}} \frac{Q^{2}}{\left|z_{0}\right|},
$$

which tends to $Q^{2} / 4 \varepsilon_{\alpha}\left|z_{0}\right|$ in the case $\varepsilon_{\alpha} \gg \varepsilon_{\beta}$. For the electrolyte, Onsager and Samaras [25] argued that the other ions give rise to a decaying factor $\exp \left(-2 \kappa\left|z_{0}\right|\right)$, where $\kappa$ is the Debye-Hückel wave number in the bulk phase $\alpha$ and the image potential should be changed to

$$
\Phi_{\alpha}\left(z_{0}\right)=\frac{\varepsilon_{\alpha}-\varepsilon_{\beta}}{2\left(\varepsilon_{\alpha}+\varepsilon_{\beta}\right) \varepsilon_{\alpha}} \frac{Q^{2}}{\left|z_{0}\right|} e^{-2 \kappa\left|z_{0}\right|} .
$$

(ii) Second, we place a charge in the less polar phase $\beta\left(z_{0}>0\right)$. This situation is relevant when there are appreciable ions in the phase $\beta$ or for not very small ion reduction factor in Eq. (4.10). Exchange of $\alpha$ and $\beta$ in Eq. (B4) gives the image potential in the phase $\beta$,

$$
\Phi_{\beta}\left(z_{0}\right)=-\frac{\varepsilon_{\alpha}-\varepsilon_{\beta}}{2\left(\varepsilon_{\alpha}+\varepsilon_{\beta}\right) \varepsilon_{\beta}} \frac{Q^{2}}{z_{0}},
$$

which is attractive to the interface and tends to $-Q^{2} / 4 \varepsilon_{\beta} z_{0}$ in the case $\varepsilon_{\alpha} \gg \varepsilon_{\beta}$. The coefficient in Eq. (B5) is larger than that in Eq. (B4) by the factor $\varepsilon_{\alpha} / \varepsilon_{\beta}$, which can be very large. However, Eq. (B5) should be much reduced in the presence of a solvation shell composed of polar molecules of the species $A$ [12-14]. See the discussion below Eq. (2.20) for the background of this picture. In electrostatics [49], a polar spherical layer enclosing a charge weakens the electric field acting on the charge by the factor $3 \varepsilon_{\beta} /\left(2 \varepsilon_{\beta}+\varepsilon_{\mathrm{A}}^{\prime}\right)$, leading to

$$
\Phi_{\beta}\left(z_{0}\right)=-\frac{\varepsilon_{\alpha}-\varepsilon_{\beta}}{2\left(\varepsilon_{\alpha}+\varepsilon_{\beta}\right) \varepsilon_{\beta}} \frac{3 \varepsilon_{\beta}}{2 \varepsilon_{\beta}+\varepsilon_{\mathrm{A}}^{\prime}} \frac{Q^{2}}{z_{0}},
$$

where $\varepsilon_{\mathrm{A}}^{\prime}\left(\sim \varepsilon_{\mathrm{A}}\right)$ is the effective dielectric constant in the layer. The coefficient in Eq. (B6) is of the same order as that in Eq. (B3) in the case $\varepsilon_{\alpha} \sim \varepsilon_{\mathrm{A}}^{\prime} \sim \varepsilon_{\mathrm{A}} \gg \varepsilon_{\beta}$.

(iii) Third, we should recognize that an image potential $\Phi_{I}$ arises generally when the dielectric constant $\varepsilon$ varies in space. We demonstrate it near the critical point, where the deviation $\delta \varepsilon=\varepsilon-\varepsilon_{c}$ from its critical value $\varepsilon_{c}$ is small. Neglecting the boundary effect, we solve $\nabla \cdot \varepsilon \nabla \Psi=-4 \pi Q \delta(r$ $\left.-\boldsymbol{r}_{0}\right)$ in powers of $\delta \varepsilon$. The deviation $\delta \Psi=\Psi-Q / \varepsilon_{c}\left|\boldsymbol{r}-\boldsymbol{r}_{0}\right|$ reads

$$
\delta \Psi\left(\boldsymbol{r}, \boldsymbol{r}_{0}\right)=\frac{Q}{4 \pi \varepsilon_{c}^{2}} \int d \boldsymbol{r}^{\prime} \frac{\boldsymbol{\nabla}^{\prime} \delta \varepsilon\left(\boldsymbol{r}^{\prime}\right)}{\left|\boldsymbol{r}-\boldsymbol{r}^{\prime}\right|} \cdot \nabla^{\prime} \frac{1}{\left|\boldsymbol{r}^{\prime}-\boldsymbol{r}_{0}\right|}
$$

to linear order in $\delta \varepsilon$. Taking the limit $\boldsymbol{r} \rightarrow \boldsymbol{r}_{0}$, we find the image potential,

$$
\Phi_{\mathrm{I}}\left(\boldsymbol{r}_{0}\right)=\frac{Q^{2}}{8 \pi \varepsilon_{c}^{2}} \int d \boldsymbol{r}^{\prime} \boldsymbol{\nabla}^{\prime} \delta \varepsilon\left(\boldsymbol{r}^{\prime}\right) \cdot \nabla^{\prime} \frac{1}{\left|\boldsymbol{r}^{\prime}-\boldsymbol{r}_{0}\right|^{2}} .
$$

In particular, if a planar interface with thickness $\xi$ is located at $z \cong 0, \varepsilon(z)$ depends only on $z$ and the integrations over $x^{\prime}$ and $y^{\prime}$ are performed to give 


$$
\Phi_{\mathrm{I}}\left(z_{0}\right)=\frac{Q^{2}}{4 \varepsilon_{c}^{2}} \int d z^{\prime} \frac{1}{z_{0}-z^{\prime}} \frac{d}{d z^{\prime}} \delta \varepsilon\left(z^{\prime}\right),
$$

where we use $\int d x \int d y\left(x^{2}+y^{2}+z^{2}\right)^{-2}=\pi / z^{2}$. For $\left|z_{0}\right| \gg \xi$, the above expression becomes

$$
\Phi_{\mathrm{I}}\left(z_{0}\right)=-\frac{\varepsilon_{\alpha}-\varepsilon_{\beta}}{4 \varepsilon_{c}^{2}} \frac{Q^{2}}{z_{0}},
$$

which is applicable both in phases $\alpha$ and $\beta$. In the electrolyte, the two functions in Eq. (B7), $1 /\left|\boldsymbol{r}^{\prime}-\boldsymbol{r}\right|$ and $1 /\left|\boldsymbol{r}^{\prime}-\boldsymbol{r}_{0}\right|$,

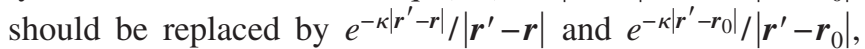
respectively, resulting in Eq. (3.7). See the discussion on the choice of $\kappa$ below Eq. (3.7).

In passing, we note that the electric potential in a finite system can be expressed in terms of the Green's function satisfying $\nabla^{2} G\left(\boldsymbol{r}, \boldsymbol{r}^{\prime}\right)=-4 \pi \delta\left(\boldsymbol{r}-\boldsymbol{r}^{\prime}\right)$ under the given boundary conditions. At fixed surface charges, its derivative with respect to $z$ vanishes at $z=0$ and $L$ in the geometry of Fig. 1, where $G\left(\boldsymbol{r}, \boldsymbol{r}^{\prime}\right)=G\left(z, z^{\prime}, \mathbf{s}\right)$ with $\mathbf{s}=\left(x-x^{\prime}, y-y^{\prime}\right)$. Its Fourier transformation $G_{k}\left(z, z^{\prime}\right)=\int d \mathbf{s} G\left(z, z^{\prime}, \mathbf{s}\right) e^{i \mathbf{k} \cdot \mathbf{s}}$ is calculated as

$$
\begin{aligned}
G_{k}\left(z, z^{\prime}\right)= & \frac{2 \pi}{k} e^{-k\left|z-z^{\prime}\right|}+\frac{4 \pi}{k\left(\sigma^{2}-1\right)}\left[\cosh \left[k\left(z-z^{\prime}\right)\right]\right. \\
& \left.+\sigma \cosh \left[k\left(z+z^{\prime}-L\right)\right]\right],
\end{aligned}
$$

where $\sigma=e^{k L}$. It is obvious that $1 /\left|\boldsymbol{r}^{\prime}-\boldsymbol{r}_{0}\right|^{2}$ in Eq. (B8) should be replaced by $G\left(\boldsymbol{r}^{\prime}, \boldsymbol{r}_{0}\right)^{2}$.

\section{APPENDIX C: DIMENSIONLESS EQUATIONS}

We write down the dimensionless equilibrium equations in one-dimensional cases. Here, the $z$ coordinate is measured in units of $a$. Using Eq. (2.14), we rewrite Eq. (2.27) as

$$
\begin{aligned}
\frac{v_{0} h}{k_{B} T}= & \ln \left[\frac{1+2 \psi}{1-2 \psi}\right]-2 \chi \psi-\chi \psi^{\prime \prime}-\frac{\hat{\varepsilon}_{1}}{32 A} U^{\prime 2}-\left(g_{1} c_{1}+g_{2} c_{2}\right) \\
& +A \hat{\varepsilon}_{1} \hat{I} c .
\end{aligned}
$$

For $Z_{1}=Z_{2}=1$, Eq. (2.30) becomes

$$
\begin{aligned}
& c_{1}=c_{1}^{0} \exp \left[g_{1} \psi-U-A \hat{\varepsilon}_{1} \hat{I} \psi\right], \\
& c_{2}=c_{2}^{0} \exp \left[g_{2} \psi+U-A \hat{\varepsilon}_{1} \hat{I} \psi\right],
\end{aligned}
$$

where $\hat{\varepsilon}_{1}=\varepsilon_{1} / \varepsilon_{c}, A$ is defined by Eq. (3.3), $\psi^{\prime \prime}=d^{2} \psi / d z^{2}$, and $U^{\prime}=d U / d z$. The $v_{0} h / k_{B} T, c_{1}^{0}$, and $c_{2}^{0}$ are constants in equilibrium. Here, $\hat{I}$ is the linear operator arising from the image interaction

$$
(\hat{I} \psi)(z)=\int \frac{d z^{\prime}}{\pi} \frac{e^{-2 \kappa a\left|z-z^{\prime}\right|}}{z-z^{\prime}} \frac{d \psi\left(z^{\prime}\right)}{d z^{\prime}} .
$$

From Eq. (2.4), we obtain the equation for $U$,

$$
-\frac{d}{d z}\left[1+\hat{\varepsilon}_{1} \psi\right] \frac{d}{d z} U=16 A\left(c_{1}-c_{2}\right) .
$$

[1] Electrostatic Effects in Soft Matter and Biophysics, edited by C. Holm, P. Kekicheff, and R. Podgornik, NATO Science Series II (Kluwer Academic, Dordrecht, 2001), Vol. 46.

[2] Y. Levin and M. E. Fisher, Physica A 225, 164 (1996); Y. Levin, Rep. Prog. Phys. 65, 1577 (2002).

[3] J. N. Israelachvili, Intermolecular and Surface Forces (Academic Press, London, 1991).

[4] Y. Marcus, Ion Solvation (Wiley, New York, 1985).

[5] P. B. Balbuena, K. P. Johnston, and P. J. Rossky, J. Phys. Chem. 100, 2706 (1996).

[6] P. Jungwirth and D. J. Trobias, J. Phys. Chem. B 105, 10468 (2001); 106, 6361 (2002).

[7] M. Born, Z. Phys. 1, 45 (1920). The right-hand side of Eq. (1.1) is simply the integration of the electrostatic energy $\varepsilon E(r)^{2} / 8 \pi$ in the region $R_{\text {ion }}^{i}<r<\infty$, where $E(r)=-Z e / \varepsilon r^{2}$ is the electric field at distance $r$ from the ion center.

[8] Y. Marcus, Chem. Rev. (Washington, D.C.) 88, 1475 (1988).

[9] D. P. Fernandez, A. R. H. Goodwin, E. W. Lemmon, J. M. H. Levet Sengers, and R. C. Williams, J. Phys. Chem. Ref. Data 26, 1125 (1997).

[10] P. Debye and K. Kleboth, J. Chem. Phys. 42, 3155 (1965).

[11] The free energy needed for electrostriction decreases with increasing the background composition $\phi$, of the more polar component. This fact is particularly important in ion-induced nucleation, see Ref. [22].

[12] M. H. Abraham, J. Liszi, and L. Meszaros, J. Chem. Phys. 70, 2491 (1979).
[13] L. Sandberg and O. Edholm, J. Chem. Phys. 116, 2936 (2002).

[14] T. Osakai and K. Ebina, J. Phys. Chem. B 102, 5691 (1998).

[15] M. Gros, S. Gromb, and C. Gavach, J. Electroanal. Chem. Interfacial Electrochem. 89, 29 (1978).

[16] J. Koryta, Electrochim. Acta 24, 293 (1979); , 29, 445 (1984).

[17] Le Quoc Hung, J. Electroanal. Chem. Interfacial Electrochem. 115, 159 (1980).

[18] T. Kakiuchi, Anal. Chem. 68, 3658 (1996).

[19] H. Jensen, V. Devaud, J. Josserand, and H. H. Girault, J. Electroanal. Chem. 537, 77 (2002).

[20] C. T. R. Wilson, Philos. Trans. R. Soc. London, Ser. A 189 , 265 (1897).

[21] J. J. Thomson, Conduction of Electricity through Gases (Cambridge University Press, Cambridge, 1906), Sec. 92. Thomson predicted formation of a liquidlike hydration shell layer around an ion in gaseous water.

[22] H. Kitamura and A. Onuki, J. Chem. Phys. 123, 124513 (2005). One of the main results in this work is that the solvent density in the vicinity of a small ion can be of the order of a liquid density in a gaseous polar one-component fluid. In this situation, the Born formula using the dielectric constant of gas is a poor approximation.

[23] S. Durand-Vidal, J. P. Simonin, and P. Turq, Electrolytes at Interfaces (Kluwer, Dordrecht, 2000).

[24] C. Wagner, Phys. Z. 25, 474 (1924).

[25] L. Onsager and N. N. T. Samaras, J. Chem. Phys. 2, 528 (1934). 
[26] Y. Levin, J. Chem. Phys. 113, 9722 (2000).

[27] Y. Levin and J. E. Flores-Mena, Europhys. Lett. 56, 187 (2001).

[28] H. Ohshima and H. Matsubara, Colloid Polym. Sci. 282, 1044 (2002).

[29] N. Matubayasi, H. Matsuo, K. Yamamoto, S. Yamaguchi, and A. Matuzawa, J. Colloid Interface Sci. 209, 398 (1999).

[30] Wei Lun Hsin, Yu-Jane Sheng, Shi-Yow Lin, and HengKwong Tsao, Phys. Rev. E 69, 031605 (2004).

[31] S. A. Safran, Statistical Thermodynamics of Surfaces, Interfaces, and Membranes (Westview Press, 2003).

[32] A. Onuki and H. Kitamura, J. Chem. Phys. 121, 3143 (2004).

[33] A. Onuki, in Nonlinear Dielectric Phenomena in Complex Liquids, edited by S. J. Rzoska, NATO Science Series II (Kluwer Academic, Dordrecht, 2004), Vol. 157.

[34] The density of dipole pairs can increase significantly even at low ion densities when the Bjerrum length $e^{2} / \varepsilon k_{B} T$ is much longer than the molecular size $a$, see Ref. [2].

[35] If $\varepsilon_{\mathrm{A}} \gg \varepsilon_{\mathrm{B}}$, we have $\varepsilon_{1} \gg \varepsilon_{0}$ and $\varepsilon_{1} / \varepsilon_{c} \cong 1 / \phi_{c}$ with $\varepsilon_{c}$ and $\phi_{c}$ being the critical values.

[36] We may choose the potential difference $\Phi_{L}-\Phi_{0}$ as a control parameter in the geometry of Fig. 1, see Ref. [33]. Then, the surface charges are fluctuating quantities and the relevant electrostatic free energy should be $F_{e}=-\int d \boldsymbol{r} \boldsymbol{D} \cdot \boldsymbol{E} / 8 \pi$. With this choice the chemical-potential contributions in Eqs. (2.11) and (2.12) remain unchanged.

[37] A. Onuki, Phase Transition Dynamics (Cambridge University Press, Cambridge, 2002).

[38] I. Borukhov, D. Andelman, and H. Orland, Phys. Rev. Lett. 79, 435 (1997). The steric effect due to ions yields terms $C_{s}^{i} n_{i} \psi$ in the free energy density at low salt densities with $C_{s}^{i}$ $\sim 1$ for $R_{\text {shell }}^{i} \sim a$. Notice that these terms can be included into the solvation terms in Eq. (2.13).

[39] V. M. Nabutovskii, N. A. Nemov, and Yu. G. Peisakhovich,
Phys. Lett. 79, 98 (1980); Zh. Eksp. Teor. Fiz. 79, 2196 (1980) [Sov. Phys. JETP 52, 111 (1980)]; Mol. Phys. 54, 979 (1985).

[40] Here $\left(g_{i}\right)_{\mathrm{Born}}=Z_{i}^{2} \ell_{\mathrm{B} c} \varepsilon_{1} / R_{\mathrm{ion}}^{i} \varepsilon_{c}$ in terms of $\ell_{\mathrm{B} c}$ in Eq. (3.4), so $\left(g_{i}\right)_{\text {Born }} / Z_{i}^{2}$ can even be of order 100.

[41] P. G. de Gennes, Rev. Mod. Phys. 57, 827 (1985).

[42] If $g_{1}>g_{2} \gg 1 / \Delta \phi$, the distance of the maximum (minimum) point from the interface position (in the Gibbs sense) is of order $\xi \ln \left(g_{1} \Delta \phi\right)\left[\xi \ln \left(g_{2} \Delta \phi\right)\right]$ from the balance $g_{i}\left(\phi_{\alpha}-\phi\right)$ $\sim g_{i} \Delta \phi \exp \left[2\left(z-z_{\text {int }}\right) / \xi\right] \sim 1$.

[43] D. Chandler, Introduction to Modern Statistical Mechanics (Oxford University Press, Oxford, 1987).

[44] E. L. Eckfeldt and W. W. Lucasse, J. Phys. Chem. 47, 164 (1943).

[45] B. J. Hales, G. L. Bertrand, and L. G. Hepler, J. Phys. Chem. 70, 3970 (1966).

[46] V. Balevicius and H. Fuess, Phys. Chem. Chem. Phys. 1, 1507 (1999).

[47] M. A. Anisimov, J. Jacob, A. Kumar, V. A. Agayan, and J. V. Sengers, Phys. Rev. Lett. 85, 2336 (2000).

[48] We may set $\mu_{0}=f^{\prime}\left(\phi_{0 K}\right)=0$ in the zeroth order without loss of generality if we redefine $f-\mu_{0} \phi$ as $f$.

[49] L. D. Landau and E. M. Lifshitz, Electrodynamics of Continuous Media (Pergamon, New York, 1984), Vol. 8.

[50] We may choose $n_{\alpha}$ as the control parameter. Then, if $\bar{n}$ is replaced by $n_{\alpha}$, Eqs. (4.51) and (4.52) still hold asymptotically. But they are applicable only much closer to the critical point, where $n_{1 \alpha} \cong n_{1 \beta}$.

[51] Yu. Kramarenko, I. Ya. Erukhimovich, and A. R. Khokhlov, Macromol. Theory Simul. 11, 462 (2002).

[52] R. F. Kayser, Phys. Rev. Lett. 56, 1831 (1986).

[53] N. A. Denesyuk and J. P. Hansen, J. Chem. Phys. 121, 3613 (2004).

[54] A. Onuki, J. Phys. Soc. Jpn. 73, 511 (2004). 